\title{
Networks of Evolutionary Processors with Subregular Filters
}

\author{
Jürgen Dassow Florin Manea ${ }^{(A)} \quad$ Bianca Truthe \\ Otto-von-Guericke-Universität Magdeburg, Fakultät für Informatik \\ PSF 4120, D-39016 Magdeburg, Germany \\ \{dassow, manea, truthe\}@iws.cs.uni-magdeburg.de
}

\begin{abstract}
In this paper, we study networks of evolutionary processors where the filters are chosen as special regular sets. We show that the use of ordered, non-counting, power-separating, circular, and suffix-closed regular languages or languages that are combinational or reverse combinational is as powerful as the use of arbitrary regular languages. On the other hand, the use of only finite languages allows only the generation of regular languages, but not all regular languages can be generated. If only filters are used that are target sets of monoids then non-context-free languages can be generated but not all regular languages.
\end{abstract}

\section{Introduction}

A basic architecture for parallel and distributed symbolic processing, related to the Connection Machine ([11] ) as well as the Logic Flow paradigm ([7]), consists of several processors, each of them being placed in a node of a graph, which are able to handle data associated with the respective node. Each node processor modifies the local data in accordance with some predefined rules and then the modified data becomes a mobile agent and is relocated in (or, in other words, communicated to) another node of the network, following a given protocol. Only that data which is able to pass a filtering process can be communicated between nodes. The filtering process may require the data to satisfy some conditions imposed by the sending processor, by the receiving processor, or by the edge existing between these nodes (seen as a chanell of communication). All the nodes send simultaneously their data and the receiving nodes handle also simultaneously all the arriving messages, according to some strategies, see [8, 11].

Following these lines, in [6] were defined the networks of language processors, as a formal languages generating model. Similarly to the above, such a network can be viewed as a graph whose nodes contain sets of productions and, at any moment of time, a language is associated with a node. In a derivation step, any node derives from its language all possible words as its new language. In a communication step, any node sends those words that satisfy an output condition, given as a regular language, to other nodes, and any node takes those words sent by

\footnotetext{
${ }^{(A)}$ Also at: Faculty of Mathematics and Computer Science, University of Bucharest, Str. Academiei 14, RO010014 Bucharest, Romania (flmanea@fmi . unibuc.ro). The work of Florin Manea is supported by the Alexander von Humboldt Foundation.
} 
the other nodes, that satisfy an input condition, also given by a regular language. The language generated by a network of language processors consists of all (terminal) words which occur in the languages associated with a given node. Also, having a biological motivation, in [5] one considers a computing model inspired by the evolution of cell populations, which might model some properties of evolving cell communities at the syntactical level. Cells are represented by words which describe their DNA sequences. Informally, at any moment of time, the evolutionary system is described by a collection of words, where each word represents one cell. Cells belong to species and their community evolves according to mutations and division which are defined by operations on words. Only those cells are accepted as surviving (correct) ones which are represented by a word in a given set of words, called the genotype space of the species. This feature parallels with the natural process of evolution. Similar ideas may be met in other bioinspired models as tissue-like membrane systems ([13]) or models from Distributed Computing area like parallel communicating grammar systems ([14]).

In this context, in [4], the Networks of Evolutionary Processors (NEPs for short) were defined. More precisely, one considers that each node of a network may be viewed as a cell having genetic information encoded in DNA sequences which may evolve by local evolutionary events, that is point mutations (substitution, deletion or insertion of a symbol). Each node is specialized in just one of these evolutionary operations. Furthermore, the data in each node is organized in the form of multisets of words, each word appearing in an arbitrarily large number of copies, and all the copies are processed in a massive parallel manner, such that all the possible events that can take place do actually take place. Clearly, this computational process is not exactly an evolutionary process in the Darwinian sense. But the rewriting operations considered might be viewed as mutations and the filtering process might be viewed as a selection process. Recombination is missing but it was asserted that evolutionary and functional relationships between genes can be captured by taking only local mutations into consideration ([17]). The computation of a NEP is conducted just as in the case of networks of language processors: initially, the nodes contain some finite sets of words, and further, these words are processed according to the rules in each node, and then, they are communicated to the other nodes, as permitted by the filters associated with the nodes, and so on; the language generated by a NEP consists of all the words which appear in a given node, called the output node.

Results on NEPs, seen as generating devices, can be found, e. g., in [12, 4, 2, 1, 3]. In the seminal paper [4], it was shown that networks of evolutionary processors are universal in that sense that they can generate any recursively enumerable language, and that networks with six nodes and regular filters are sufficient to get all recursively enumerable languages; the result was further improved from the descriptional complexity point of view (see, e. g., [1]) and NEPs having only two types of nodes, and their computational power, were investigated in [2]. Among the same lines, we aproach in this paper several variants of NEPs where the communication protocols are restricted.

It is clear that the communication between the nodes of the network depends on two things: the shape of the network (i.e., it depends on which edges exist in the network) and the filters of the nodes. Here we discuss in detail one of these two aspects. Namely, we analyze the computational power of NEPs having the filters of all the nodes in different classes of subregular languages. In [4], and several other papers discussing NEPs as generating devices (see, e. g., [2] and [12] for an early survey), regular filters were used and the computational completeness of the model was shown. Therefore, it seems natural to study in which way the computational 
power of the model is affected when simpler filters are used. To summarize, we show that the use of ordered, non-counting, power-separating, circular, and suffix-closed regular languages or languages that are combinational or reverse combinational is as powerful as the use of arbitrary regular languages; on the other hand, the use of only finite languages allows only the generation of regular languages, but not all regular languages can be generated. If only filters are used that are target sets of monoids then non-context-free languages can be generated but not all regular languages.

\section{Definitions}

We assume that the reader is familiar with the basic concepts of formal language theory (see e. g. [15]). We here only recall some notations used in the paper.

By $V^{*}$ we denote the set of all words (strings) over $V$ (including the empty word $\lambda$ ). The length of a word $w$ is denoted by $|w|$. The number of occurrences of a letter $a$ or of letters from a set $A$ is denoted by $|w|_{a}$ and $|w|_{A}$, respectively. For the number of elements of a set $A$, we write \# $(A)$.

In the proofs we shall often add new letters of an alphabet $U$ to a given alphabet $V$. In all these situations, we assume that $V \cap U=\emptyset$.

A phrase structure grammar is specified as a quadruple

$$
G=(N, T, P, S)
$$

where $N$ is a set of non-terminals, $T$ is a set of terminals, $P$ is a finite set of productions which are written as $\alpha \rightarrow \beta$ with $\alpha \in(N \cup T)^{+} \backslash T^{*}$ and $\beta \in(N \cup T)^{*}$, and $S \in N$ is the axiom.

A grammar $G=(N, T, P, S)$ is in Geffert normal form ([9]) if the set of nonterminals consists of the axiom $S$ and three more letters $A, B, C$ only and all rules in $P$ have the form

$$
A B C \rightarrow \lambda \quad \text { or } \quad S \rightarrow v \text { with } v \in(N \cup T)^{*}
$$

By FIN, REG, CF, CS, and RE we denote the families of finite, regular, context-free, context-sensitive, and recursively enumerable languages, respectively. For a language $L$ over $V$, we set

$$
\begin{aligned}
\operatorname{Suf}(L) & =\left\{y \mid x y \in L \text { for some } x \in V^{*}\right\} \\
\operatorname{Comm}(L) & =\left\{a_{i_{1}} \ldots a_{i_{n}} \mid a_{1} \ldots a_{n} \in L, n \geq 1,\left\{i_{1}, i_{2}, \ldots, i_{n}\right\}=\{1,2, \ldots, n\}\right\}, \\
\operatorname{Circ}(L) & =\left\{a_{i+1} a_{i+2} \ldots a_{n} a_{1} a_{2} \ldots a_{i} \mid n \geq 1,1 \leq i \leq n, a_{1} a_{2} \ldots a_{n} \in L\right\} .
\end{aligned}
$$

It is known that $\operatorname{Suf}(L)$ and $\operatorname{Circ}(L)$ are regular for a regular language $L$.

We consider the following restrictions for regular languages. Let $L$ be a language and $V=\operatorname{alph}(L)$ the minimal alphabet of $L$. We say that $L$ is

- combinational iff it can be represented in the form $L=V^{*} A$ for some subset $A \subseteq V$,

- bi-combinational iff it can be represented in the form $L=V^{*} A$ or $L=A V^{*}$ for some subset $A \subseteq V$,

- definite iff it can be represented in the form $L=A \cup V^{*} B$ where $A$ and $B$ are finite subsets of $V^{*}$, 
- nilpotent iff $L$ is finite or $V^{*} \backslash L$ is finite,

- commutative iff $L=\operatorname{Comm}(L)$,

- circular iff $L=\operatorname{Circ}(L)$,

- suffix-closed (or fully initial or multiple-entry language) iff $x y \in L$ for some $x, y \in V^{*}$ implies $y \in L$ (or equivalently, $\operatorname{Suf}(L)=L$ ),

- non-counting (or star-free) iff there is an integer $k \geq 1$ such that, for any $x, y, z \in V^{*}$, $x y^{k} z \in L$ if and only if $x y^{k+1} z \in L$,

- power-separating iff for any $x \in V^{*}$ there is a natural number $m \geq 1$ such that either $J_{x}^{m} \cap L=\emptyset$ or $J_{x}^{m} \subseteq L$ where $J_{x}^{m}=\left\{x^{n} \mid n \geq m\right\}$,

- ordered iff $L$ is accepted by some finite automaton $\mathcal{A}=\left(Z, V, \delta, z_{0}, F\right)$ where $(Z, \preceq)$ is a totally ordered set and, for any $a \in V, z \preceq z^{\prime}$ implies $\delta(z, a) \preceq \delta\left(z^{\prime}, a\right)$.

It is obvious that combinational, definite, nilpotent, and ordered languages are regular, whereas non-regular languages of the other types mentioned above exist.

By $C O M B, B C O M B, D E F, N I L, C O M M, C I R C, S U F, N C, P S$, and ORD we denote the families of all combinational, definite, nilpotent, regular commutative, regular circular, regular suffix-closed, regular non-counting, regular power-separating, and ordered languages, respectively. The relations between these language families are investigated e.g. in [10] and [18] and can be given by Figure 1. Moreover, we add the family $M O N$ of all languages of the form $V^{*}$, where $V$ is an alphabet (languages of $M O N$ are target sets of monoids; we call them monoidal languages).

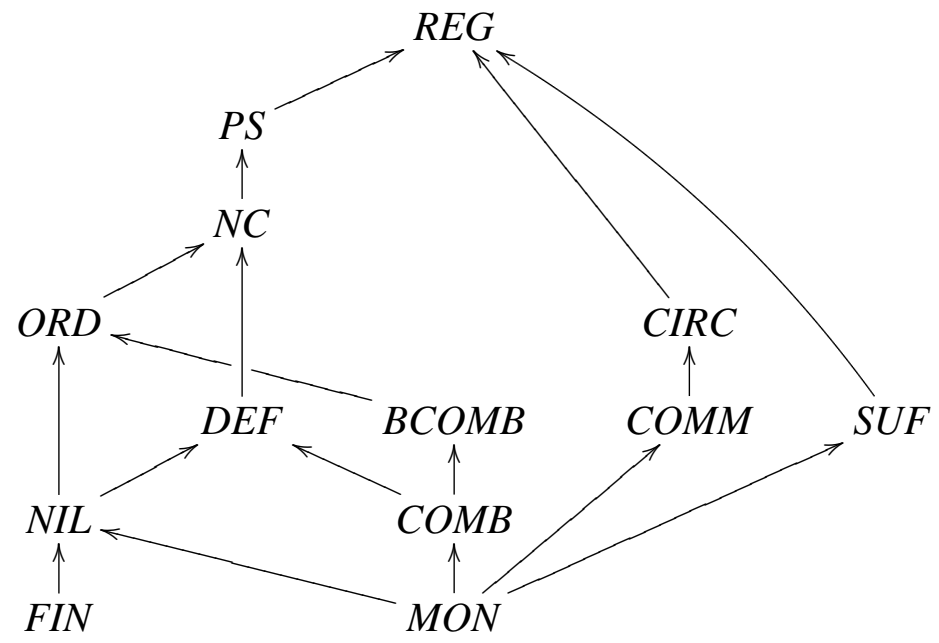

Figure 1: Hierarchy of subregular languages (an arrow from $X$ to $Y$ denotes $X \subset Y$ ( $X$ is a proper subset of $Y$ ), and if two families are not connected by a directed path then they are incomparable)

Let $V=\left\{a_{1}, a_{2}, \ldots, a_{n}\right\}$ be an alphabet with a fixed order and $\psi: V^{*} \longrightarrow \mathbb{N}_{0}^{n}$ be the Parikh mapping with $\psi(w)=\left(|w|_{a_{1}},|w|_{a_{2}}, \ldots,|w|_{a_{n}}\right)$. The mapping $\psi$ is extended to languages such that the Parikh image of a language $L$ is $\psi(L)=\{\psi(w) \mid w \in L\}$. Two languages $L$ and $L^{\prime}$ are called letter-equivalent if $\psi(L)=\psi\left(L^{\prime}\right)$ (if for any word from one language there is a word in the other language which has the same number of each letter of the alphabet). By $\psi^{-1}$, we denote the mapping that assigns to a tuple $p=\left(k_{1}, k_{2}, \ldots, k_{n}\right) \in \mathbb{N}_{0}^{n}$ the set of all words $w$ with $p$ as the Parikh vector:

$$
\psi^{-1}(p)=\left\{\left.w|| w\right|_{a_{1}}=k_{1},|w|_{a_{2}}=k_{2}, \ldots,|w|_{a_{n}}=k_{n}\right\} .
$$


The extension to sets of $n$-tuples yields, for a set $M$, the set $\psi^{-1}(M)=\{w \mid \psi(w) \in M\}$.

For a language $L$, we have $\psi(L)=\psi(\operatorname{Comm}(L))$ and $\psi^{-1}(\psi(L))=\operatorname{Comm}(L)$.

We call a production $\alpha \rightarrow \beta$ a

- substitution if $|\alpha|=|\beta|=1$,

- deletion if $|\alpha|=1$ and $\beta=\lambda$.

We introduce insertion as a counterpart of deletion. We write $\lambda \rightarrow a$, where $a$ is a letter. The application of an insertion $\lambda \rightarrow a$ derives from a word $w$ any word $w_{1} a w_{2}$ with $w=w_{1} w_{2}$ for some (possibly empty) words $w_{1}$ and $w_{2}$.

The productions are applied like context-free rewriting rules. We say that a word $v$ derives a word $w$, written as $v \Longrightarrow w$, if there are words $x, y$ and a production $\alpha \rightarrow \beta$ such that $v=x \alpha y$ and $w=x \beta y$. If the rule $p$ applied is important, we write $v \Longrightarrow_{p} w$.

We now introduce the basic concept of this paper, the networks of evolutionary processors (NEPs for short).

Definition 2.1 Let $X$ be a family of regular languages.

(i) A network of evolutionary processors (of size $n$ ) with filters of the set $X$ is a tuple

$$
\mathcal{N}=\left(V, N_{1}, N_{2}, \ldots, N_{n}, E, j\right)
$$

where

- $V$ is a finite alphabet,

- for $1 \leq i \leq n, N_{i}=\left(M_{i}, A_{i}, I_{i}, O_{i}\right)$ where

- $M_{i}$ is a set of evolutionary rules of a certain type, i. e.,

$M_{i} \subseteq\{a \rightarrow b \mid a, b \in V\}, M_{i} \subseteq\{a \rightarrow \lambda \mid a \in V\}$, or $M_{i} \subseteq\{\lambda \rightarrow b \mid b \in V\}$,

- $A_{i}$ is a finite subset of $V^{*}$,

- $I_{i}$ and $O_{i}$ are languages from $X$ over $V$,

- E is a subset of $\{1,2, \ldots, n\} \times\{1,2, \ldots, n\}$, and

- $j$ is a natural number such that $1 \leq j \leq n$.

(ii) A configuration $C$ of $\mathcal{N}$ is an $n$-tuple $C=(C(1), C(2), \ldots, C(n))$ where $C(i)$ is a subset of $V^{*}$ for $1 \leq i \leq n$.

(iii) Let $C=(C(1), C(2), \ldots, C(n))$ and $C^{\prime}=\left(C^{\prime}(1), C^{\prime}(2), \ldots, C^{\prime}(n)\right)$ be two configurations of $\mathcal{N}$. We say that $C$ derives $C^{\prime}$ in one

- evolutionary step (written as $C \Longrightarrow C^{\prime}$ ) if, for $1 \leq i \leq n, C^{\prime}(i)$ consists of all words $w \in C(i)$ to which no rule of $M_{i}$ is applicable and of all words $w$ for which there are a word $v \in C(i)$ and a rule $p \in M_{i}$ such that $v \Longrightarrow_{p} w$ holds,

- communication step (written as $C \vdash C^{\prime}$ ) if, for $1 \leq i \leq n$,

$$
C^{\prime}(i)=\left(C(i) \backslash O_{i}\right) \cup \bigcup_{(k, i) \in E}\left(C(k) \cap O_{k} \cap I_{i}\right) .
$$

The computation of $\mathcal{N}$ is a sequence of configurations $C_{t}=\left(C_{t}(1), C_{t}(2), \ldots, C_{t}(n)\right)$, $t \geq 0$, such that

- $C_{0}=\left(A_{1}, A_{2}, \ldots, A_{n}\right)$,

- for any $t \geq 0, C_{2 t}$ derives $C_{2 t+1}$ in one evolutionary step: $C_{2 t} \Longrightarrow C_{2 t+1}$,

- for any $t \geq 0, C_{2 t+1}$ derives $C_{2 t+2}$ in one communication step: $C_{2 t+1} \vdash C_{2 t+2}$. 
(iv) The language $L(\mathcal{N})$ generated by $\mathcal{N}$ is defined as

$$
L(\mathcal{N})=\bigcup_{t \geq 0} C_{t}(j)
$$

where $C_{t}=\left(C_{t}(1), C_{t}(2), \ldots, C_{t}(n)\right), t \geq 0$ is the computation of $\mathcal{N}$.

Intuitively, a network with evolutionary processors is a graph consisting of some, say $n$, nodes $N_{1}, N_{2}, \ldots, N_{n}$ (called processors) and the set of edges given by $E$ such that there is a directed edge from $N_{k}$ to $N_{i}$ if and only if $(k, i) \in E$. Any processor $N_{i}$ consists of a set of evolutionary rules $M_{i}$, a set of words $A_{i}$, an input filter $I_{i}$ and an output filter $O_{i}$. We say that $N_{i}$ is a substitution node or a deletion node or an insertion node if $M_{i} \subseteq\{a \rightarrow b \mid a, b \in V\}$ or $M_{i} \subseteq\{a \rightarrow \lambda \mid a \in V\}$ or $M_{i} \subseteq\{\lambda \rightarrow b \mid b \in V\}$, respectively. The input filter $I_{i}$ and the output filter $O_{i}$ control the words which are allowed to enter and to leave the node, respectively. With any node $N_{i}$ and any time moment $t \geq 0$ we associate a set $C_{t}(i)$ of words (the words contained in the node at time $t$ ). Initially, $N_{i}$ contains the words of $A_{i}$. In an evolutionary step, we derive from $C_{t}(i)$ all words applying rules from the set $M_{i}$. In a communication step, any processor $N_{i}$ sends out all words $C_{t}(i) \cap O_{i}$ (which pass the output filter) to all processors to which a directed edge exists (only the words from $C_{t}(i) \backslash O_{i}$ remain in the set associated with $N_{i}$ ) and, moreover, it receives from any processor $N_{k}$ such that there is an edge from $N_{k}$ to $N_{i}$ all words sent by $N_{k}$ and passing the input filter $I_{i}$ of $N_{i}$, i. e., the processor $N_{i}$ gets in addition all words of $C_{t}(k) \cap O_{k} \cap I_{i}$. We start with an evolutionary step and then communication steps and evolutionary steps are alternately performed. The language consists of all words which are in the node $N_{j}$ (also called the output node, $j$ is chosen in advance) at some moment $t, t \geq 0$.

For a family $X \subseteq R E G$, we denote the family of languages generated by networks with evolutionary processors where all filters are of type $X$ by $\mathcal{E}(X)$.

The following fact is obvious.

Lemma 2.2 Let $X, Y$ be subfamilies of REG such that $X \subseteq Y$. Then $\mathcal{E}(X) \subseteq \mathcal{E}(Y)$.

The following theorem is known (see, e. g., [4]]).

Theorem $2.3 \mathcal{E}(R E G)=R E$.

\section{Results}

Let $\mathcal{N}=\left(V, N_{1}, N_{2}, \ldots, N_{n}, E, j\right)$ be a network with evolutionary processors. In some cases, it is useful to require that the output node $N_{j}$ has the properties $A_{j}=\emptyset, M_{j}=\emptyset$, and there is no edge leaving $N_{j}$. If $O_{j}=\emptyset$ then a possibly leaving edge can be removed without further effect. With these properties, a word belongs to the generated language $L(\mathcal{N})$ if and only if it is communicated at some time to $N_{j}$. The node $N_{j}$ itself does not contribute to the whole language, neither by its own initial language nor by modifying words. Additionally, words that have arrived in $N_{j}$ will not be recycled. We now show that, for each NEP, there is an euqivalent NEP with this property. 
Theorem 3.1 For every network $\mathcal{N}$ of evolutionary processors, there is a network $\mathcal{N}^{\prime}$ of evolutionary processors that generates the same language as $\mathcal{N}$ and has the property that its output node $N^{\prime}$ has the form $N^{\prime}=\left(\emptyset, \emptyset, I^{\prime}, O^{\prime}\right)$ for some regular languages $I^{\prime}, O^{\prime}$ over the network's working alphabet and no edge is leaving $N^{\prime}$.

Proof. Let $\mathcal{N}=\left(V, N_{1}, N_{2}, \ldots, N_{n}, E, j\right)$ be a network of evolutionary processors where the output node $N_{j}$ has not the required property: $N_{j} \neq\left(\emptyset, \emptyset, I_{j}, O_{j}\right)$ for any sets $I_{j}, O_{j}$ or there is an edge leaving node $N_{j}$. We define a new network $\mathcal{N}^{\prime}=\left(V, N_{1}^{\prime}, N_{2}^{\prime}, \ldots, N_{n+4}^{\prime}, E^{\prime}, n+4\right)$ by

$$
\begin{aligned}
N_{i}^{\prime}= & N_{i} \text { for } 1 \leq i \leq n, \\
N_{i}^{\prime}= & \left(M_{i}, \emptyset, I_{i}, O_{i}\right) \text { for } n+1 \leq i \leq n+4, \\
E^{\prime}= & E \cup\{(i, n+1) \mid(i, j) \in E\} \\
& \cup\{(n+1, n+2),(n+1, n+4),(n+2, n+3),(n+2, n+4),(n+3, n+2)\}
\end{aligned}
$$

where

$$
\begin{aligned}
& M_{n+1}=\emptyset, \quad M_{n+2}=M_{j}, \quad M_{n+3}=\emptyset, \quad M_{n+4}=\emptyset, \\
& A_{n+1}=A_{j}, \quad A_{n+2}=\emptyset, \quad A_{n+3}=\emptyset, \quad A_{n+4}=\emptyset \text {, } \\
& I_{n+1}=I_{j}, \quad I_{n+2}=V^{*}, \quad I_{n+3}=V^{*} \backslash O_{j}, \quad I_{n+4}=V^{*} \text {, } \\
& O_{n+1}=V^{*}, \quad O_{n+2}=V^{*}, \quad O_{n+3}=V^{*}, \quad O_{n+4}=V^{*} \text {. }
\end{aligned}
$$

The network is illustrated below:

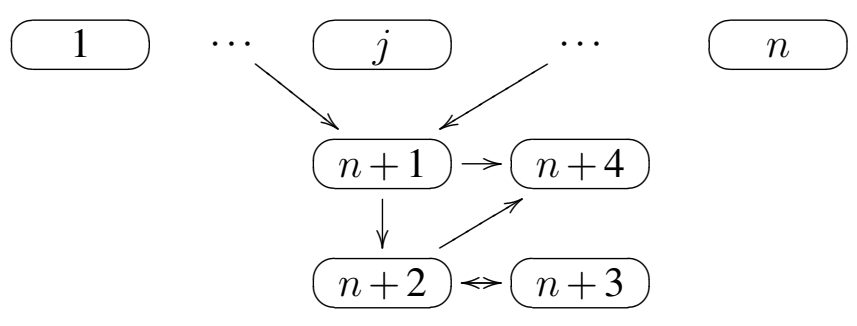

The new output node $N_{n+4}^{\prime}$ satisfies the condition because $N_{n+4}^{\prime}=\left(\emptyset, \emptyset, V^{*}, V^{*}\right)$ and no edge leaves the node $N_{n+4}^{\prime}$. We now show that $L\left(\mathcal{N}^{\prime}\right)=L(\mathcal{N})$.

The subnetwork consisting of $N_{1}^{\prime}, N_{2}^{\prime}, \ldots, N_{n}^{\prime}$ is the same as $\mathcal{N}$. The initial sets of $N_{j}^{\prime}$ and $N_{n+1}^{\prime}$ as well as the input filters and incoming edges coincide. Hence, if a word $w$ is in $N_{j}$ at an even moment $t$, then $w$ is also in this moment in node $N_{j}^{\prime}$ and $N_{n+1}^{\prime}$. The word is then sent unchanged to the output node $N_{n+4}^{\prime}$. Thus, $w \in L(\mathcal{N})$ and $w \in L\left(\mathcal{N}^{\prime}\right)$. Additionally, $w$ is also sent to $N_{n+2}^{\prime}$ where the same rules as in $N_{j}$ can be applied. Hence, if a word $v$ is derived in $N_{j}$ (and, hence, $v \in L(\mathcal{N})$ ) then $v$ is derived in $N_{n+2}^{\prime}$ and will be sent to the output node in the next communication step, hence, $v \in L\left(\mathcal{N}^{\prime}\right)$. If the word $v$ remains in $N_{j}$ then a word $u \in L(\mathcal{N})$ will be derived from $v$ in $N_{j}$. In $\mathcal{N}^{\prime}$, the word $v$ will also be sent to $N_{n+3}^{\prime}$ which takes the word and sends it back to $N_{n+2}^{\prime}$ where it will be derived to $u$ which will be sent to the output node afterwards. Hence, as long as a word is modified in $N_{j}$, the same word is modified in $N_{n+2}^{\prime}$ with intermediate communication to $N_{n+3}^{\prime}$ and all these words also arrive in the output node. Thus, $L(\mathcal{N}) \subseteq L\left(\mathcal{N}^{\prime}\right)$.

Every word $w \in L\left(\mathcal{N}^{\prime}\right)$ came to node $N_{n+4}^{\prime}$ from node $N_{n+1}^{\prime}$ or $N_{n+2}^{\prime}$. If it came from $N_{n+1}^{\prime}$ then the word was also in node $N_{j}$, hence, $w \in L(\mathcal{N})$. If it came from $N_{n+2}^{\prime}$ then it has been 
derived from a word $v$ which came from $N_{n+1}^{\prime}$ or $N_{n+3}^{\prime}$. If $v$ came from $N_{n+1}^{\prime}$ then $v$ was also in $N_{j}$ and has derived $w$, hence, $w \in L(\mathcal{N})$. If $v$ came from $N_{n+3}^{\prime}$ then $v$ was previously in node $N_{n+2}^{\prime}$ and was derived from a word $u$. Furthermore, $v \notin O_{j}$. If $u$ came from $N_{n+1}^{\prime}$ then $u$ was also in $N_{j}$ and has derived $v$ which remained there and derived $w$, hence, $w \in L(\mathcal{N})$. If $u$ came from $N_{n+3}^{\prime}$ then the argumentation can be repeated because for every word in $u$ in $N_{n+2}^{\prime}$ there was a word $\tilde{u}$ in $N_{n+1}^{\prime}$ with $\tilde{u} \Longrightarrow_{M_{j}}^{*} u$ and all words during this derivation did not belong to $O_{j}$. Hence, $\tilde{u}$ was also in $N_{j}$ where the same derivation of $u$ took place. Thus, we have the inclusion $L\left(\mathcal{N}^{\prime}\right) \subseteq L(\mathcal{N})$.

Since $L\left(\mathcal{N}^{\prime}\right)=L(\mathcal{N})$ the network $\mathcal{N}^{\prime}$ has the required properties.

Networks of evolutionary processors with regular filters that are suffix-closed or circular can generate all recursively enumerable languages.

Theorem $3.2 \mathcal{E}(S U F)=R E$.

Proof. Let $L$ be a recursively enumerable language. Let $\mathcal{N}=\left(V, N_{1}, N_{2}, \ldots, N_{n}, E, j\right)$ be a network with evolutionary processors and filters from $R E G$ such that $L(\mathcal{N})=L$. For any node $N_{i}=\left(M_{i}, A_{i}, I_{i}, O_{i}\right)$, we construct the sets

$$
\begin{aligned}
I_{i}^{\prime} & =\{X\} I_{i}\{Y\} \cup \operatorname{Suf}\left(I_{i}\right)\{Y\} \cup\{\lambda\}, \\
O_{i}^{\prime} & =\{X\} O_{i}\{Y\} \cup \operatorname{Suf}\left(O_{i}\right)\{Y\} \cup\{\lambda\},
\end{aligned}
$$

where $X$ and $Y$ are two new symbols. By definition, $I_{i}^{\prime}$ and $O_{i}^{\prime}$ are suffix-closed. We assume that the network $\mathcal{N}$ has the property $N_{j}=\left(\emptyset, \emptyset, I_{j}, O_{j}\right)$ and no edge leaves the output node (cf. Theorem 3.1).

We consider the network

$$
\mathcal{N}^{\prime}=\left(V \cup\{X, Y\}, N_{1}^{\prime}, N_{2}^{\prime}, \ldots, N_{n}^{\prime}, N_{n+1}^{\prime}, N_{n+2}^{\prime}, E^{\prime}, n+2\right)
$$

with

$$
\begin{aligned}
N_{i}^{\prime} & =\left(M_{i},\{X\} A_{i}\{Y\}, I_{i}^{\prime}, O_{i}^{\prime}\right) \text { for } 1 \leq i \leq n, \\
N_{n+1}^{\prime} & =\left(\{X \rightarrow \lambda, Y \rightarrow \lambda\}, \emptyset, I_{j}^{\prime}, V^{*}\right), \\
N_{n+2}^{\prime} & =\left(\emptyset, \emptyset, V^{*}, \emptyset\right), \\
E^{\prime} & =E \cup\{(i, n+1) \mid(i, j) \in E\} \cup\{(n+1, n+2)\} .
\end{aligned}
$$

It is obvious that the filters of $N_{n+1}^{\prime}$ and $N_{n+2}^{\prime}$ are suffix-closed, too. Thus $\mathcal{N}^{\prime}$ is a network of type $S U F$.

We now prove that $L(\mathcal{N})=L\left(\mathcal{N}^{\prime}\right)$. We start with words of the form $X w Y$ and as long as these words are changed according to rules of $M_{i}, 1 \leq i \leq n$, they can only be sent to nodes $N_{s}^{\prime}$ with $1 \leq s \leq n$ and $N_{n+1}^{\prime}$. Thus we simulate a derivation in $\mathcal{N}$ (in $\mathcal{N}^{\prime}$ we have an $X$ in front of and a $Y$ behind the word $w$ occurring in $\mathcal{N}$ ) and get into $N_{n+1}^{\prime}$ exactly those words $X w Y$ whose subword $w$ comes into $N_{j}$. Now $X$ and $Y$ are removed and the resulting word $w$ is sent to $N_{n+2}^{\prime}$. Other words cannot arrive in $N_{n+2}^{\prime}$ and other words do not appear in $N_{j}$. Hence, we have the equality $L\left(\mathcal{N}^{\prime}\right)=L(\mathcal{N})$. 
Theorem $3.3 \mathcal{E}(C I R C)=R E$.

Proof. We repeat the proof of Theorem 3.2 with the following modifications. We set

$$
I_{i}^{\prime}=\operatorname{Circ}\left(\{X\} I_{i}\{Y\}\right) \text { and } O_{i}^{\prime}=\operatorname{Circ}\left(\{X\} O_{i}\{Y\}\right) \text { for } 1 \leq i \leq n .
$$

This ensures that $\operatorname{Circ}(F)=F$ for all filters $F$ of the new network $\mathcal{N}^{\prime}$. Then the proof proceeds as in the case of suffix-closed filters.

The smallest subfamilies of the family of regular languages we consider are the family of the finite languages $(F I N)$ and the family of all languages of the form $V^{*}$ for an alphabet $V$ $(M O N)$. Every such language can be generated by a NEP where all filters belong to one class given in Figure 1.

Theorem 3.4 For all families $X$ from Figure 1 the inclusion $F I N \subset \mathcal{E}(X)$ holds.

Proof. According to Lemma 2.2, we have, for all families $X$ from Figure 1, the inclusion

$$
\mathcal{E}(F I N) \subseteq \mathcal{E}(X) \quad \text { or } \quad \mathcal{E}(M O N) \subseteq \mathcal{E}(X)
$$

Hence, we only have to show that $F I N \subset \mathcal{E}(F I N)$ and $F I N \subset \mathcal{E}(M O N)$. Then the theorem follows from these inclusions.

Let $L$ be a finite set over $V$. Then the networks

$$
\mathcal{N}_{1}^{F I N}=(V,(\emptyset, L, \emptyset, \emptyset), \emptyset, 1) \quad \text { and } \quad \mathcal{N}_{1}^{M O N}=\left(V,\left(\emptyset, L, \emptyset^{*}, \emptyset^{*}\right), \emptyset, 1\right)
$$

with all filters from either $F I N$ or $M O N$ generate $L$. Thus, any finite language belongs to $\mathcal{E}(F I N)$ and $\mathcal{E}(M O N)$.

The networks

$$
\mathcal{N}_{2}^{F I N}=(\{a\},(\{\lambda \rightarrow a\},\{\lambda\}, \emptyset, \emptyset), \emptyset, 1) \quad \text { and } \quad \mathcal{N}_{2}^{M O N}=\left(\{a\},\left(\{\lambda \rightarrow a\},\{\lambda\}, \emptyset^{*}, \emptyset^{*}\right), \emptyset, 1\right)
$$

with filters from $F I N$ or $M O N$ generate the infinite language $\{a\}^{*}$. Hence, FIN is a proper subset of $\mathcal{E}(X)$ for $X \in\{F I N, M O N\}$.

Theorem 3.5 For all families $X$ from Figure 1 the inclusion $M O N \subset \mathcal{E}(X)$ holds.

Proof. According to Lemma 2.2, we have, for all families $X$ from Figure 1, the inclusion

$$
\mathcal{E}(F I N) \subseteq \mathcal{E}(X) \quad \text { or } \quad \mathcal{E}(M O N) \subseteq \mathcal{E}(X)
$$

Hence, we only have to show that $M O N \subset \mathcal{E}(F I N)$ and $M O N \subset \mathcal{E}(M O N)$. Then the theorem follows from these inclusions.

Let $V$ be an alphabet and $L=V^{*}$. Then the networks

$$
\begin{aligned}
\mathcal{N}_{1}^{F I N} & =(V,(\{\lambda \rightarrow a \mid a \in V\},\{\lambda\}, \emptyset, \emptyset), \emptyset, 1) \text { and } \\
\mathcal{N}_{1}^{M O N} & =\left(V,\left(\{\lambda \rightarrow a \mid a \in V\},\{\lambda\}, \emptyset^{*}, \emptyset^{*}\right), \emptyset, 1\right)
\end{aligned}
$$

with all filters from either FIN or MON generate $L$. Thus, any language $L=V^{*}$ belongs to $\mathcal{E}(F I N)$ and $\mathcal{E}(M O N)$. 
According to Theorem 3.4, $\mathcal{E}(F I N)$ also contains all finite languages. They cannot be expressed in the form $V^{*}$. Hence, $M O N \subset \mathcal{E}(F I N)$.

The network

$$
\mathcal{N}_{2}=\left(\{a, b\},\left(\{\lambda \rightarrow b\},\{a\}, \emptyset^{*}, \emptyset^{*}\right), \emptyset, 1\right)
$$

generates the language of all words over $\{a, b\}$ with exactly one occurrence of $a$ which is not expressible as $V^{*}$. Hence, $M O N \subset \mathcal{E}(M O N)$.

We now show that also in more general cases the inclusion

$$
X \subseteq \mathcal{E}(X)
$$

holds.

Theorem 3.6 Let $X$ be a class mentioned in Figure 1 except FIN. Each language $L \in X$ can be generated by a NEP $\mathcal{N}$ with exactly two nodes and with filters from type $X$ only.

Proof. Let $X$ be a class mentioned in Figure 1 except $F I N$. Then $M O N \subseteq X$. Furthermore, let $L \in X$ be a language over an alphabet $V$. We construct a NEP

$$
\mathcal{N}=\left(V, N_{1}, N_{2},\{(1,1),(1,2)\}, 2\right)
$$

with $N_{1}=\left(M_{1}, A_{1}, I_{1}, O_{1}\right)$ and $N_{2}=\left(M_{2}, A_{2}, I_{2}, O_{2}\right)$ which is defined as follows:

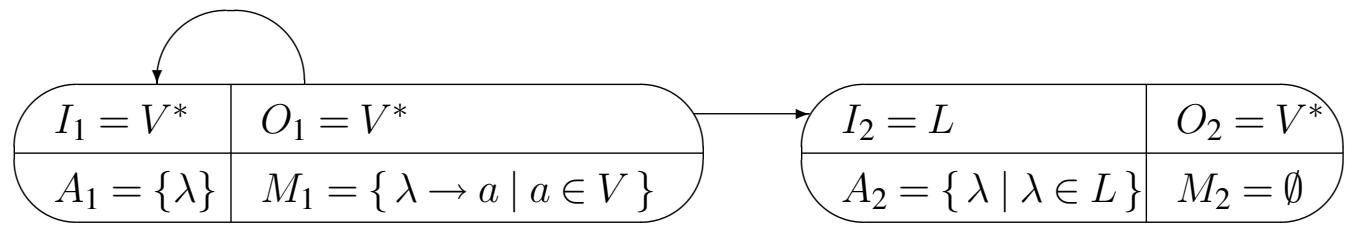

Every word $w \in V^{+}$will be derived in node $N_{1}$ and be communicated to node $N_{2}$ which accepts all words that also belong to $L$. The language generated by $\mathcal{N}$ is $L(\mathcal{N})=A_{2} \cup\left(V^{+} \cap L\right)=L$. All filters are of type $X$.

If one restricts the networks to be without loops (as usual in the literature, e.g., [1]), the previous statement also holds (just insert another node that only forwards the words it receives from $N_{1}$ to $N_{1}$ ).

Together with Theorem 3.4, the previous theorem yields the following statement.

Corollary 3.7 For each class $X$ mentioned in Figure 1, we have $X \subseteq \mathcal{E}(X)$.

In the following theorems, we give some results on the set $\mathcal{E}(F I N)$. 


\section{Theorem $3.8 \mathcal{E}(F I N) \subset R E G$.}

Proof. Let $\mathcal{N}=\left(V, N_{1}, N_{2}, \ldots, N_{n}, E, j\right)$ be a network with finite filters. Obviously, a word $w$ is in $N_{j}$ if and only if it is in $A_{j}$ or satisfies $I_{j}$ or is obtained from a word in $N_{j}$ by application of a rule in $M_{j}$. We set

$$
U=\left\{a \mid \lambda \rightarrow a \in M_{j}\right\}, V^{\prime}=\left\{a^{\prime} \mid a \in V\right\}, \text { and } U^{\prime}=\left\{a^{\prime} \mid a \in U\right\} .
$$

Let $h:\left(V \cup V^{\prime}\right)^{*} \rightarrow V^{*}$ be the homomorphism defined by

$$
h(a)=a \text { for } a \in V \quad \text { and } \quad h\left(a^{\prime}\right)= \begin{cases}\lambda, & \text { for } a^{\prime} \in U^{\prime} \\ a, & \text { for } a^{\prime} \in V^{\prime} \backslash U^{\prime}\end{cases}
$$

and $\tau:\left(V \cup V^{\prime}\right)^{*} \rightarrow V^{*}$ be the finite substitution where $\tau(a)=\tau\left(a^{\prime}\right)$ for $a \in V$ and $\tau(a)$ consists of all $b \in V \cup\{\lambda\}$ such that there are an integers $s \geq 0$ and $b_{0}, b_{1}, \ldots, b_{s-1} \in V$ and $b_{s} \in V \cup\{\lambda\}$ such that $a=b_{0}, b=b_{s}$, and $b_{i} \rightarrow b_{i+1} \in M_{j}$ for $0 \leq i \leq s-1$ (note that $s=0$ implies $a=b$ ). Furthermore, let

$$
k=\max \left\{|w| \mid w \in O_{j} \cup I_{j} \cup A_{j}\right\}+1 .
$$

We note the following facts:

- Assume that there is a word $w$ of length at least $k$ in $L(\mathcal{N})$. Then $w$ is in $C_{t}(j)$ for some $t$. By its length, it cannot leave the node, and thus all words which have a length at least $k$ and can be obtained by application of rules of $M_{j}$ to $w$ belong to $L(\mathcal{N})$, too.

- If $w$ with $|w| \geq k+1$ is in $L(\mathcal{N})$, then $w$ is obtained from a word $v \in L(\mathcal{N})$ of length $k$ by application of rules in $M_{j}$ (since substitutions and deletions do not increase the length, the shortest words in $L(\mathcal{N})$ with length at least $k$ are obtained by an insertion from a word of length less than $k$ and thus they have length $k$ ).

Now it is easy to see that

$$
L(\mathcal{N})=\left(L(\mathcal{N}) \cap \bigcup_{i=0}^{k-1} V^{i}\right) \cup\left(\tau\left(h^{-1}\left(L(\mathcal{N}) \cap V^{k}\right)\right) \cap \bigcup_{i \geq k} V^{i}\right)
$$

holds. Since finite languages are regular and regular languages are closed under inverse homomorphisms, finite substitutions, intersection, and union, $L(\mathcal{N})$ is regular. Hence $\mathcal{E}(F I N) \subseteq R E G$ holds.

Let $L=\left\{a^{n} b^{m} \mid n \geq 1, m \geq 1\right\}$. Assume that a network $\mathcal{N}=\left(V, N_{1}, N_{2}, \ldots, N_{n}, E, j\right)$ with finite filters exists such that $L(\mathcal{N})=L$. Let $k=\max \left\{|z| \mid z \in O_{j} \cup I_{j} \cup A_{j}\right\}$ and $w=a^{r} b^{s}$ for some $r, s \geq k$. Let $w \in C_{t}(j)$. By definition of $k$, the word $w$ is not in $I_{j}$ and $A_{j}$. Thus there is a word $v \in C_{t-1}(j)$ with $v \Longrightarrow w$ by application of a rule $p$ of $M_{j}$. We distinguish some cases.

Case 1. $p=\lambda \rightarrow a$. Then $v=a^{r-1} b^{s}$. The word $v$ can also be derived to $a^{r-1} b^{s} a \in C_{t}(j)$ and therefore $a^{r-1} b^{s} a \in L(\mathcal{N})$. However, $a^{r-1} b^{s} a \notin L$. Hence, we have a contradiction.

Case 2. $p=\lambda \rightarrow b$. Then $v=a^{r} b^{s-1}$ and $a^{r} b^{s-1} \Longrightarrow b a^{r} b^{s-1} \in C_{t}(j) \subseteq L(\mathcal{N})=L$ in contrast to $b a^{r} b^{s-1} \notin L$ by definition of $L$.

Case 3. $p=X \rightarrow x$ for some $X \in V \backslash\{a, b\}$ and $x \in\{a, b, \lambda\}$. Then $v=v^{\prime} X v^{\prime \prime}$ for two words $v^{\prime}, v^{\prime \prime}$ and $v \in C_{t-1}(j) \subseteq L(\mathcal{N})=L$. But $v^{\prime} X v^{\prime \prime} \notin L$ by its definition. 
Case 4. $p=a \rightarrow b$. If $v=a^{r} b^{p} a b^{q}$ with $p+q=s-1$ and $p \geq 1$, we get a contradiction as in

Case 3. If $v=a^{r+1} b^{s-1}$ (the rule is applied to the last occurrence of $a$ to obtain $w$ ), we can also derive $b a^{r} b^{s-1}$ and obtain a contradiction as in Case 2.

Case 5. $p=b \rightarrow a$. As in Case 4 , we can obtain a contradiction.

Case 6. $p=a \rightarrow \lambda$. Now we get the derivation

$$
w=a^{r} b^{s} \Longrightarrow a^{r-1} b^{s} \Longrightarrow a^{r-2} b^{s} \Longrightarrow \cdots \Longrightarrow b^{s} .
$$

Since all words $a^{q} b^{s}$ with $q \geq 1$ are not in $O_{j}$ by the definition of $k$ and $s \geq k$, the words remain in node $N_{j}$ if a communication step is done. Therefore $b^{s} \in C_{t+2 r}(j) \subseteq L(\mathcal{N})$ in contrast to $b^{s} \notin L$.

Case 7. $p=b \rightarrow \lambda$. As in Case 6 we can derive a contradiction.

Therefore, our assumption has to be false, which proves $L \notin \mathcal{E}(F I N)$. Because $L$ is regular, the inclusion $\mathcal{E}(F I N) \subseteq R E G$ is proper.

Lemma 3.9 For each $N E P \mathcal{N}$ with only finite filters, we can construct a $N E P \mathcal{N}^{\prime}$ with only one processor and finite filters that generates the same language as $\mathcal{N}$.

Proof. Let $\mathcal{N}=\left(V, N_{1}, N_{2}, \ldots, N_{n}, E, j\right)$ be a NEP with finite filters. Let $B_{j}$ be the set of all words that enter some time the node $N_{j}$ :

$$
\begin{aligned}
B_{j} & =\left\{w \in V^{*} \mid \exists t \exists i:(i, j) \in E \text { and } w \in C_{2 t+1}(i) \cap O_{i} \cap I_{j}\right\} \\
& =\left\{w \in V^{*} \mid \exists i:(i, j) \in E \text { and } w \in O_{i} \cap I_{j} \cap \bigcup_{t \geq 0} C_{2 t+1}(i)\right\} .
\end{aligned}
$$

The set $B_{j}$ is finite $\left(B_{j} \subseteq I_{j}\right)$ and can be computed since the set $L^{\prime}(i)=\bigcup_{t \geq 0} C_{2 t+1}(i)$ is regular (see proof of Theorem 3.8, the equation also holds for $L^{\prime}(i)$ instead of $L(\mathcal{N})$ ).

Let $\mathcal{N}^{\prime}=\left(V, N_{1}^{\prime}, \emptyset, 1\right)$ the NEP with the processor $N_{1}^{\prime}=\left(M_{j}, A_{j} \cup B_{j}, \emptyset, O_{j}\right)$. Let $C$ and $C^{\prime}$ be the configurations of $N$ and $N^{\prime}$, respectively. We show inductively that every word $w$ which is in node $N_{j}$ at a time $2 t^{\prime}$ or $2 t^{\prime}+1$ (for a $t^{\prime} \geq 0$ ) is also in node $N_{1}^{\prime}$ at a time $2 t^{\prime \prime}$ or $2 t^{\prime \prime}+1$ (for a $\left.t^{\prime \prime} \geq 0\right)$, respectively, and vice versa.

- $w \in C_{0}(j)$. Then $w \in A_{j}$ and therefore $w \in C_{0}^{\prime}(1)$.

- $w \in C_{2 t^{\prime}+1}(j)$ with $t^{\prime} \geq 0$. Then

(a) $w \in C_{2 t^{\prime}}(j)$ and $M_{j}$ is not applicable or

(b) there is a word $v \in C_{2 t^{\prime}}(j)$ which yields $w\left(v \Longrightarrow_{M_{j}} w\right)$.

In Case (a), we have $w \in C_{2 t^{\prime \prime}}^{\prime \prime}(1)$ for a $t^{\prime \prime} \geq 0$ by induction hypothesis. Since no rule is applicable, we also have $w \in C_{2 t^{\prime \prime}+1}^{\prime}(1)$. In Case (b), we have $v \in C_{2 t^{\prime \prime}}^{\prime}(1)$ for a $t^{\prime \prime} \geq 0$ by induction hypothesis. Since $v \Longrightarrow_{M_{j}} w$ it is $w \in C_{2 t^{\prime \prime}+1}^{\prime}(1)$.

- $w \in C_{2 t^{\prime}}(j)$ with $t^{\prime} \geq 1$. Then

(a) there is $k$ with $1 \leq k \leq n,(k, j) \in E$, and $w \in C_{2 t^{\prime}-1}(k) \cap O_{k} \cap I_{j}$ or

(b) $w \in C_{2 t^{\prime}-1}(j) \backslash O_{j}$. 
In Case (a), we have $w \in B_{j}$ and therefore $w \in C_{0}^{\prime}(1)$. In Case (b), we have $w \in C_{2 t^{\prime \prime}+1}^{\prime}(1)$ for a $t^{\prime \prime} \geq 0$ by induction hypothesis. Since $w \notin O_{j}$ we also have $w \in C_{2 t^{\prime \prime}+2}^{\prime}(1)$.

- $w \in C_{0}^{\prime}(1)$. Then
(a) $w \in A_{j}$ or
(b) $w \in B_{j}$.

In Case (a), we also have $w \in C_{0}(j)$. In Case (b), we have $w \in C_{2 t^{\prime}}(j)$ for a $t^{\prime} \geq 1$.

- $w \in C_{2 t^{\prime \prime}+1}^{\prime}(1)$ with $t^{\prime \prime} \geq 0$. Then

(a) $w \in C_{2 t^{\prime \prime}}^{\prime \prime}(1)$ and $M_{j}$ is not applicable or

(b) there is a word $v \in C_{2 t^{\prime \prime}}^{\prime}(1)$ which yields $w\left(v \Longrightarrow_{M_{j}} w\right)$.

In Case (a), we have $w \in C_{2 t^{\prime}}(j)$ for a $t^{\prime} \geq 0$ by induction hypothesis. Since no rule is applicable, we also have $w \in C_{2 t^{\prime}+1}(j)$. In Case (b), we have $v \in C_{2 t^{\prime}}(j)$ for a $t^{\prime} \geq 0$ by induction hypothesis. Since $v \Longrightarrow_{M_{j}} w$ it is $w \in C_{2 t^{\prime}+1}(1)$.

- $w \in C_{2 t^{\prime \prime}}^{\prime}(1)$ with $t^{\prime \prime} \geq 1$. Then $w \in C_{2 t^{\prime \prime}-1}^{\prime}(1) \backslash O_{j}$ (because there is no 'real' communication). We have $w \in C_{2 t^{\prime}+1}(j)$ for a $t^{\prime} \geq 0$ by induction hypothesis. Since $w \notin O_{j}$ we also have $w \in C_{2 t^{\prime}+2}(j)$.

By this induction, it is shown that $L\left(\mathcal{N}^{\prime}\right)=L(\mathcal{N})$.

Let $\mathcal{N}=\left(V, N_{1}, \emptyset, 1\right)$ be a NEP with only finite filters and $N_{1}=(M, A, \emptyset, O)$. If $N_{1}$ is a deleting or substituting processor then the language generated is finite ([2]). Let now $N_{1}$ be an inserting processor. Furthermore, let $U=\{a \mid \lambda \rightarrow a \in M\}$ be the set of all letters that can be inserted. If $O=\emptyset$ then the language generated is

$$
L(\mathcal{N})=\left\{u_{1} x_{1} u_{2} x_{2} \cdots u_{n} x_{n} u_{n+1} \mid x_{1} x_{2} \cdots x_{n} \in A \text { and } u_{i} \in U^{*} \text { for } 1 \leq i \leq n+1\right\} .
$$

In the case that $O \neq \emptyset$, let $\min _{O}=\min \{|w| \mid w \in O\}, \max _{O}=\max \{|w| \mid w \in O\}$, and

$$
\begin{gathered}
A_{1}=\left\{w \mid w=u_{1} x_{1} u_{2} x_{2} \cdots u_{n} x_{n} u_{n+1}, x_{1} x_{2} \cdots x_{n} \in A \text { and } u_{i} \in U^{*}\right. \\
\text { for } \left.1 \leq i \leq n+1, \text { and }|w|<\min _{O}\right\}, \\
A_{2}=\left\{w \mid w=u_{1} x_{1} u_{2} x_{2} \cdots u_{n} x_{n} u_{n+1}, x_{1} x_{2} \cdots x_{n} \in A \text { and } u_{i} \in U^{*}\right. \\
\text { for } \left.1 \leq i \leq n+1, \text { and }|w|=\min _{O}\right\}, \\
A_{3}=\left\{w \mid w=u_{1} x_{1} u_{2} x_{2} \cdots u_{n} x_{n} u_{n+1}, x_{1} x_{2} \cdots x_{n} \in A_{2} \backslash O \text { and } u_{i} \in U^{*}\right. \\
\text { for } \left.1 \leq i \leq n+1, \text { and }|w|=\min _{O}+1\right\}, \\
A_{4}=\left\{w \mid w=u_{1} x_{1} u_{2} x_{2} \cdots u_{n} x_{n} u_{n+1}, x_{1} x_{2} \cdots x_{n} \in A_{3} \backslash O \text { and } u_{i} \in U^{*}\right. \\
\text { for } \left.1 \leq i \leq n+1, \text { and }|w|=\min _{O}+2\right\}, \\
A_{\Delta+4}=\left\{w \mid w=u_{1} x_{1} u_{2} x_{2} \cdots u_{n} x_{n} u_{n+1}, x_{1} x_{2} \cdots x_{n} \in A_{\Delta+3} \backslash O \text { and } u_{i} \in U^{*}\right. \\
\text { for } \left.1 \leq i \leq n+1, \text { and }|w|=\max _{O}+1\right\}, \\
A_{\Delta+5}=\left\{w \mid w=u_{1} x_{1} u_{2} x_{2} \cdots u_{n} x_{n} u_{n+1}, x_{1} x_{2} \cdots x_{n} \in A_{\Delta+4} \text { and } u_{i} \in U^{*}\right. \\
\text { for } \left.1 \leq i \leq n+1, \text { and }|w|>\max _{O}+1\right\}
\end{gathered}
$$


with $\Delta=\left(\max _{O}+1\right)-\left(\min _{O}+2\right)=\max _{O}-\min _{O}-1$.

Then the language generated is

$$
L(\mathcal{N})=\bigcup_{i=1}^{\Delta+5} A_{i}
$$

Theorem 3.10 $\mathcal{E}(F I N) \subset \mathcal{E}(N I L)$.

Proof. The inclusion $\mathcal{E}(F I N) \subseteq \mathcal{E}(N I L)$ follows from Lemma 2.2.

Let $V=\{a\}$ and $L=\{a\} \cup\left\{a^{n} \mid n \geq 3\right\}$. The complement of $L$ is $\bar{L}=\{\lambda, a a\}$ which is finite. Hence, $L$ is a nilpotent language. By Corollary 3.7, $L \in \mathcal{E}(N I L)$.

Suppose $L$ is generated by a network with only finite filters. Then, by Lemma 3.9, there is a network $\mathcal{N}$ with only one node $N=(M, A, \emptyset, O)$ that generates $L$. Since $L$ is infinite, this node must be inserting. Hence, the rule set is $M=\{\lambda \rightarrow a\}$. If the initial set $A$ contains $\lambda$ then $\lambda \in L(\mathcal{N})$ which is in contrast to $\lambda \notin L$. If the initial set $A$ contains $a$ or $a a$ then the word a a belongs to the generated language $L(\mathcal{N})$ which is in contrast to $a a \notin L$. If the initial set only contains words $a^{n}$ with $n \geq 3$ then the word $a$ is not generatable but $a \in L$ which is a contradiction, too. Hence, there is no network with only finite filters that generates $L$. Thus, we have $L \in \mathcal{E}(N I L) \backslash \mathcal{E}(F I N)$.

Let $\mathcal{N}=\left(V, N_{1}, N_{2}, \ldots, N_{n}, E, j\right)$ be a network of evolutionary filters where all filters and all initial languages $A_{i}$ for $1 \leq i \leq n$ are commutative. Then, for each node $N_{i}$ with $1 \leq i \leq n$ and each time $t \geq 0$, the set $C_{t}(i)$ is commutative, too. Hence, the generated language $L(\mathcal{N})$ is also commutative.

We next show that only commutative filters are not sufficient to generate all recursively enumerable languages.

\section{Theorem 3.11 $\mathcal{E}(C O M M) \subset \mathcal{E}(C I R C)$.}

Proof. The inclusion $\mathcal{E}(C O M M) \subseteq \mathcal{E}(C I R C)$ follows from Lemma 2.2.

Let $L=\left\{a^{n} b^{n} \mid n \geq 1\right\}$. According to Theorem 3.3, we have $L \in \mathcal{E}(C I R C)$. Let us assume that $L \in \mathcal{E}(C O M M)$. Then there is a NEP $\mathcal{N}$ which has only commutative filters and which generates the language $L$. Since $L$ is infinite and networks with only substitution and deletion nodes always generate finite languages, $\mathcal{N}$ contains an inserting processor. The number of as is unbounded (for each natural number $n$, there is a word $w \in L$ with more than $n$ occurrences of $a$ ). Hence, there are a natural number $s \geq 0$ and letters $x_{0}, x_{1}, \ldots, x_{s}$ with $x_{s}=a$ such that the network contains the rules $\lambda \rightarrow x_{0}$ and $x_{i} \rightarrow x_{i+1}$ for $0 \leq i \leq s-1$ and there is a word $w_{1} a w_{2} \in L$ which is derived from a word $v_{1} v_{2}$ by applying these rules (possibly not only these rules), starting with the insertion of $x_{0}$ between $v_{1}$ and $v_{2}$. Instead, $x_{0}$ could also be inserted at the end of $v_{1} v_{2}$. All words derived from $v_{1} x_{0} v_{2}$ are letter equivalent to those derived from $v_{1} v_{2} x_{0}$. Thus, if a word derived from $v_{1} x_{0} v_{2}$ can pass a filter then also a word that is derived from $v_{1} v_{2} x_{0}$ in the same manner can pass that filter. Hence, in the same way how $w_{1} a w_{2}$ is derived and communicated to the output node, also the word $w_{1} w_{2} a$ is derived and communicated to the output node. But $w_{1} w_{2} a \notin L$. Thus the language $L$ cannot be generated by a NEP where all filters are commutative. This implies $L \notin \mathcal{E}(C O M M)$ and the proper inclusion $\mathcal{E}(C O M M) \subset \mathcal{E}(C I R C)$ is shown.

We now continue with investigations on the class $\mathcal{E}(M O N)$. 
Theorem 3.12 Let $L$ be a semi-linear language. Then $\operatorname{Comm}(L) \in \mathcal{E}(M O N)$.

Proof. For each semi-linear language $L$, a regular grammar $G$ can be constructed which generates a language that is letter-equivalent to $L$, i. e., $\psi(L(G))=\psi(L)([[16])$. Then we have

$$
\psi^{-1}(\psi(L(G)))=\psi^{-1}(\psi(L))
$$

and therefore $\operatorname{Comm}(L(G))=\operatorname{Comm}(L)$. Thus, for any semi-linear language $L$, a regular grammar $G$ can be constructed with $\operatorname{Comm}(L(G))=\operatorname{Comm}(L)$. We now give, for a regular gram$\operatorname{mar} G$, a network with only monoidal filters that generates the language $\operatorname{Comm}(L(G))$. Then we have a method to construct, for a semi-linear language $L$, a network with only monoidal filters that generates the language $\operatorname{Comm}(L)$.

Let $G=(N, T, P, S)$ be a regular grammar where all rules in $P$ have the form $A \rightarrow a B$ or $A \rightarrow a$ for nonterminals $A, B \in N$ and a terminal symbol $a \in T$. Additionally, the rule $S \rightarrow \lambda$ is permitted if the axiom $S$ does not occur on the right hand side of a rule.

For each nonterminal $X \in N$, we set

$$
R(X)=\{\langle a Y\rangle \mid X \rightarrow a Y \in P\}
$$

as the set of symbols representing the right hand sides of the nonterminating rules,

$$
T_{t}(X)=\{a \mid a \in T \text { and } X \rightarrow a \in P\}
$$

as the set of all terminal symbols that are generated by $X$ with terminating,

$$
N_{\text {post }}(X)=\{Y \mid Y \in N \text { and } \exists a \in T: X \rightarrow a Y \in P\}
$$

as the set of all nonterminals that are generated by $X$, and

$$
T_{\text {pre }}(X)=\{a \mid a \in T \text { and } \exists Y \in N: Y \rightarrow a X \in P\}
$$

as the set of all terminal symbols that are generated at the same time as $X$. The terminating nonterminals (those nonterminals $X \in N$ for which a rule $X \rightarrow a \in P$ exists for a terminal symbol $a \in T$ ) are gathered in the set $N_{t}$. Furthermore, we set $R=\{\langle a Y\rangle \mid \exists X \in N: X \rightarrow a Y \in P\}$ and $\hat{T}=T \cup R$.

For every nonterminal $X \in N$, we define two nodes

$$
N_{X}=\left(M_{X}, A_{X}, I_{X}, O_{X}\right) \quad \text { and } \quad N_{X^{\prime}}=\left(M_{X^{\prime}}, A_{X^{\prime}}, I_{X^{\prime}}, O_{X^{\prime}}\right)
$$

as follows:

$$
\begin{aligned}
M_{X} & =\{\lambda \rightarrow\langle a Y\rangle \mid X \rightarrow a Y \in P\}, & M_{X^{\prime}} & =\left\{\langle a X\rangle \rightarrow a \mid a \in T_{\text {pre }}(X)\right\}, \\
A_{X} & =\emptyset, & A_{X^{\prime}} & = \begin{cases}\{\lambda\}, & \text { if } X=S, \\
\emptyset, & \text { otherwise },\end{cases} \\
I_{X} & =T^{*}, & I_{X^{\prime}} & =\left(\left\{\langle a X\rangle \mid a \in T_{\text {pre }}(X)\right\} \cup T\right)^{*}, \\
O_{X} & =\hat{T}^{*}, & O_{X^{\prime}} & =T^{*} .
\end{aligned}
$$

For each terminating nonterminal $X \in N_{t}$, we additionally define a node

$$
N_{X_{t}}=\left(M_{X_{t}}, A_{X_{t}}, I_{X_{t}}, O_{X_{t}}\right)
$$


by $M_{X_{t}}=\left\{\lambda \rightarrow a \mid a \in T_{t}(X)\right\}, A_{X_{t}}=\emptyset$, and $I_{X_{t}}=O_{X_{t}}=T^{*}$.

The output node $N_{\text {out }}=\left(M_{\text {out }}, A_{\text {out }}, I_{\text {out }}, O_{\text {out }}\right)$ which is responsible for the language generated is defined by $M_{\text {out }}=A_{\text {out }}=\emptyset$ and $I_{\text {out }}=O_{\text {out }}=T^{*}$.

The edges of the network will be as follows: there is an edge from node $N_{X}$ to node $N_{Y^{\prime}}$ if $Y \in N_{\text {post }}(X)$, an edge from node $N_{X^{\prime}}$ to node $N_{X}$ for each nonterminal $X \in N$, an edge from $N_{X^{\prime}}$ to $N_{X_{t}}$ for each terminating nonterminal $X \in N_{t}$, an edge from $N_{X_{t}}$ to $N_{\text {out }}$ for each terminating nonterminal $X \in N_{t}$, and if the rule $S \rightarrow \lambda$ exists in $P$ then there is an edge from node $N_{S^{\prime}}$ to node $N_{\text {out }}$ :

$$
\begin{aligned}
& E=\left\{\left(X, Y^{\prime} \mid Y \in N_{\text {post }}(X)\right\} \cup\left\{\left(X^{\prime}, X\right) \mid X \in N\right\} \cup\left\{\left(X^{\prime}, X_{t}\right) \mid X \in N_{t}\right\}\right. \\
& \cup\left\{\left(X_{t}, \text { out }\right) \mid X \in N_{t}\right\} \cup E_{\lambda}
\end{aligned}
$$

with

$$
E_{\lambda}= \begin{cases}\left\{\left(S^{\prime}, \text { out }\right)\right\}, & \text { if } S \rightarrow \lambda \in P \\ \emptyset, & \text { otherwise }\end{cases}
$$

The network with the working alphabet $\hat{T}$, the nodes described above, the edge set $E$ and the output node $N_{\text {out }}$ is denoted by $\mathcal{N}$. Note that all filters belong to the family $M O N$.

The network simulates $G$ in the sense that a terminal symbol is created somewhere in a word whenever it is appended by the grammar. The nonterminal in a sentential form gives the 'state' in which the sentential form currently is. This is represented in the network by the different nodes. The derivation in $G$ starts with the axiom $S$. The simulation starts in node $N_{S^{\prime}}$ (the only node where the initial set is not empty). In this moment, no rule can be applied. So, the empty word is sent to the node $N_{S}$ and to $N_{S_{t}}$ if there is a rule $S \rightarrow a \in P$ for a terminal symbol $a$ and to $N_{\text {out }}$ if the rule $S \rightarrow \lambda$ exists in $P$. In the latter case, we have $\lambda \in L(G)$ and $\lambda \in L(\mathcal{N})$.

For each rule $X \rightarrow a Y \in P$, the node $N_{X}$ can generate the intermediate symbol $\langle a Y\rangle$. Then the word is sent out to all nodes $N_{Z}$ (where $Z$ is on the right hand side of a rule for $X$ : $Z \in N_{\text {post }}(X)$, also if the only rule with $X$ and $Z$ is $X \rightarrow b Z$ ), but only the node $N_{Y}$ takes the word. This node changes the symbol $\langle a Y\rangle$ to $a$ and sends the word to the node $N_{Y}$ and, if $Y$ is a terminating nonterminal, also to the node $N_{Y_{t}}$.

Whenever a node $N_{X_{t}}$ receives a word $w$ then $w X$ is letter-equivalent to a word $v X$ that is a sentential form of $G$. Now every terminal symbol $a$ that can be appended to $v$ in a terminating derivation step can be inserted into the word $w$. This word is sent to the output node. Hence, it belongs to the generated language.

Thus, $L(\mathcal{N})=\left\{w \mid w \in T^{*}\right.$ and $\left.\exists v \in L(G): \psi(v)=\psi(w)\right\}=\operatorname{Comm}(L(G))$.

Corollary 3.13 There are non-context-free languages that can be generated by networks with only filters from the family MON.

Proof. Let $n$ be a natural number, $V=\left\{a_{1}, a_{2}, \ldots, a_{n}\right\}$ and $L$ be the non-context-free language $L=\left\{\left.w\left|w \in V^{*},\right| w\right|_{a_{1}}=|w|_{a_{2}}=\cdots=|w|_{a_{n}}\right\}$. This language is semi-linear and commutative. Hence, $L=\operatorname{Comm}(L)$. According to Theorem 3.12, $L \in \mathcal{E}(M O N)$.

Even some languages that are not semi-linear can be generated as the following example shows. 
Lemma 3.14 The non-semi-linear language $L=\left\{a^{2^{n}} \mid n \geq 0\right\}$ can be generated by a network with only monoidal filters.

Proof. Let $V=\{S, A, F, a\}$ and $\mathcal{N}=\left(V, N_{1}, N_{2}, N_{3}, N_{4}, N_{5}, E, 5\right)$ be the following network:

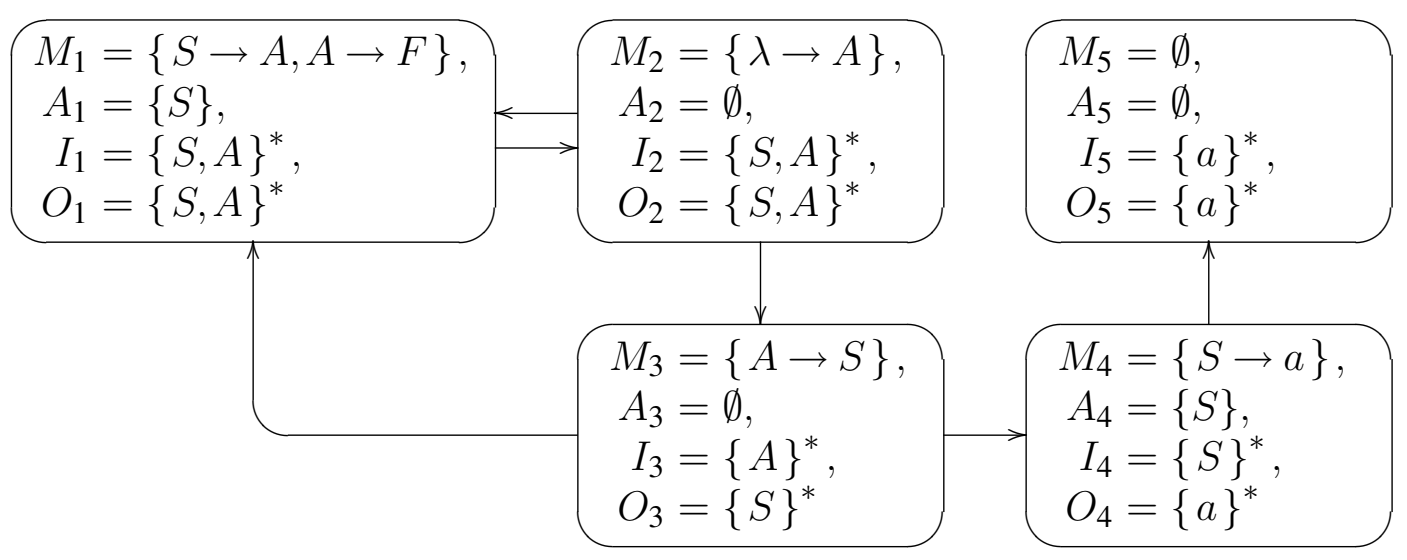

In the beginning, we have the word $S$ in node $N_{1}$. We consider a word $S^{n}$ for $n \geq 1$ in node $N_{1}$ in an even moment (in the beginning or after a communication step). One occurrence of $S$ is replaced by $A$, then the word is sent to node $N_{2}$ where another copy of $A$ is inserted. This word $w$ goes back to node $N_{1}$ and it goes on to node $N_{3}$ which takes it if no $S$ appears in the word. If in $N_{1}$ the rule $A \rightarrow F$ is applied then the symbol $F$ is introduced which cannot be replaced. Due to the output filter $O_{1}$, the word will be trapped in $N_{1}$ for ever. If in the word $w$ no $S$ is present then the only rule which can be applied is $A \rightarrow F$ and the cycle is stopped. If $w$ still contains an $S$ then it is replaced by $A$ and $N_{2}$ inserts another $A$. So, the words move between $N_{1}$ and $N_{2}$ where alternatingly an $S$ is replaced by $A$ and an $A$ is inserted until the word only contains $A$ s. The word is then $A^{n+1}$. Hence, the number of letters has been doubled.

In $N_{3}$, each $A$ is replaced by $S$. The word is $S^{n+1}$ when it leaves $N_{3}$. It moves to $N_{1}$ and to $N_{4}$. In $N_{1}$, the cycle starts again with a word $S^{m}$ for $m \geq 1$. All arriving words in $N_{4}$ have the form $S^{n}$ with $n \geq 2$. In order to cover also the case $n=1$, the initial language of this node consists of $S$. In $N_{4}$, every letter $S$ is replaced by the symbol $a$ before the word leaves to node and moves to the output node $N_{5}$.

Hence, $L(\mathcal{N})=\left\{a^{2^{n}} \mid n \geq 0\right\}$.

An upper bound for the class $\mathcal{E}(M O N)$ is given in the following theorem.

Theorem 3.15 $\mathcal{E}(M O N) \subset \mathcal{E}(C O M B)$.

Proof. The inclusion $\mathcal{E}(M O N) \subseteq \mathcal{E}(C O M B)$ follows from Lemma 2.2 .

Let $L=\left\{w b \mid w \in\{a, b\}^{*}\right\}$. The language $L$ can be written as $V^{*} B$ with $V=\{a, b\}$ and $B=\{b\}$. Hence, $L \in C O M B$ and, by Corollary 3.7, $L \in \mathcal{E}(C O M B)$.

Suppose $L \in \mathcal{E}(M O N)$. Then there is a NEP $\mathcal{N}$ which has only filters that belong to the class $M O N$ and which generates the language $L$. Since $L$ is infinite and networks with only substitution and deletion nodes generate finite languages, $\mathcal{N}$ contains an inserting processor. The number of $a$ s is unbounded (for each natural number $n$, there is a word $w \in L$ with more than $n$ occurrences of $a$ ). Hence, there are a natural number $s \geq 0$ and letters $x_{0}, x_{1}, \ldots, x_{s}$ with $x_{s}=a$ such that the network contains the rules $\lambda \rightarrow x_{0}$ and $x_{i} \rightarrow x_{i+1}$ for $0 \leq i \leq s-1$ 
and there is a word $w_{1} a w_{2} \in L$ which is derived from a word $v_{1} v_{2}$ by applying these rules (possibly not only these rules), starting with the insertion of $x_{0}$ between $v_{1}$ and $v_{2}$. Instead, $x_{0}$ could also be inserted at the end of $v_{1} v_{2}$. All words derived from $v_{1} x_{0} v_{2}$ are letter equivalent to those derived from $v_{1} v_{2} x_{0}$. Thus, if a word derived from $v_{1} x_{0} v_{2}$ can pass a filter then also a word that is derived from $v_{1} v_{2} x_{0}$ in the same manner can pass that filter. Hence, in the same way how $w_{1} a w_{2}$ is derived and communicated to the output node, also the word $w_{1} w_{2} a$ is derived and communicated to the output node. But $w_{1} w_{2} a \notin L$. Thus, the language $L$ cannot be generated by a NEP where the filters belong to $M O N$. This implies $L \notin \mathcal{E}(M O N)$ and therefore $\mathcal{E}(M O N) \subset \mathcal{E}(C O M B)$.

Corollary 3.16 The classes REG of all regular languages and $C F$ of all context-free languages are incomparable with the class $\mathcal{E}(M O N)$ of all languages generatable by networks with only monoidal filters.

Proof. The regular language

$$
L=\left\{w b \mid w \in\{a, b\}^{*}\right\}
$$

cannot be generated by a network with filters from $M O N$ only (according to the proof of Theorem 3.15. However, the non-context-free language

$$
L=\left\{\left.w\left|w \in\{a, b, c\}^{*},\right| w\right|_{a}=|w|_{b}=|w|_{c}\right\}
$$

can be generated (Corollary 3.13).

We now further investigate the class $\mathcal{E}(C O M B)$.

Theorem 3.17 $R E G \subset \mathcal{E}(C O M B)$.

Proof. We first show that a regular language can be generated by a NEP with combinational filters only. The idea is based on the network constructed in the proof of Theorem 3.12. Since we now have combinational filters, we can check whether a symbol has been appended to a word (and not only inserted at an arbitrary position). We modify the network $\mathcal{N}$ from the proof of Theorem 3.12 to a network $\mathcal{N}^{\prime}$ as follows: We introduce a new set $T^{\prime}=\left\{a^{\prime} \mid a \in T\right\}$ and set $\hat{T}=T \cup R \cup T^{\prime}$. The input filters $I_{X^{\prime}}$ are now $I_{X^{\prime}}=\hat{T}^{*}\left\{\langle a X\rangle \mid a \in T_{\text {pre }}(X)\right\}$ (the change from $T$ to $\hat{T}$ has only formal reasons because it has to be a superset of the set of the last symbols; the network ensures that there is exactly one bracketed symbol in an arriving word). The rules in the nodes $N_{X_{t}}$ representing the terminating rules are changed to $M_{X_{t}}=\left\{\lambda \rightarrow a^{\prime} \mid a \in T_{t}(X)\right\}$ (because we have to check again whether the inserted symbol was appended). Then these output filters are $O_{X_{t}}=\hat{T}^{*}$. For checking whether the last symbol was inserted at the end of the word, we introduce a new node $N_{X_{t}^{\prime}}$ (for each $X \in N_{t}$ ) with

$$
M_{X_{t}^{\prime}}=\left\{a^{\prime} \rightarrow a \mid a \in T_{t}(X)\right\}, A_{X_{t}^{\prime}}=\emptyset, I_{X_{t}^{\prime}}=\hat{T}^{*}\left\{a^{\prime} \mid a \in T_{t}(X)\right\} \text {, and } O_{X_{t}^{\prime}}=T^{*} .
$$

This node also replaces the primed symbol by its unprimed version. The network $\mathcal{N}^{\prime}$ simulates the derivation of the grammar $G$. Hence, $L\left(\mathcal{N}^{\prime}\right)=L(G)$. Thus, $R E G \subseteq \mathcal{E}(C O M B)$.

Let $L=\left\{w \mid w \in\{a, b\}^{*}\right.$ and $\left.|w|_{a}=|w|_{b}\right\}$. This language is not regular but, according to Theorem 3.12, $L \in \mathcal{E}(M O N)$ (because $L$ is semi-linear and $\operatorname{Comm}(L)=L$ ) and therefore $L \in \mathcal{E}(C O M B)$. Thus, $R E G \subset \mathcal{E}(C O M B)$. 
Lemma 3.18 The language $L=\left\{a^{n} b^{n} \mid n \geq 1\right\}$ can be generated by a a network with only combinational filters.

Proof. Let $V=\left\{a, B, B^{\prime}, B^{\prime \prime}, b\right\}$ and $\mathcal{N}=\left(V \cup\{F\}, N_{1}, N_{2}, \ldots, N_{7}, E, 7\right)$ be the following network:

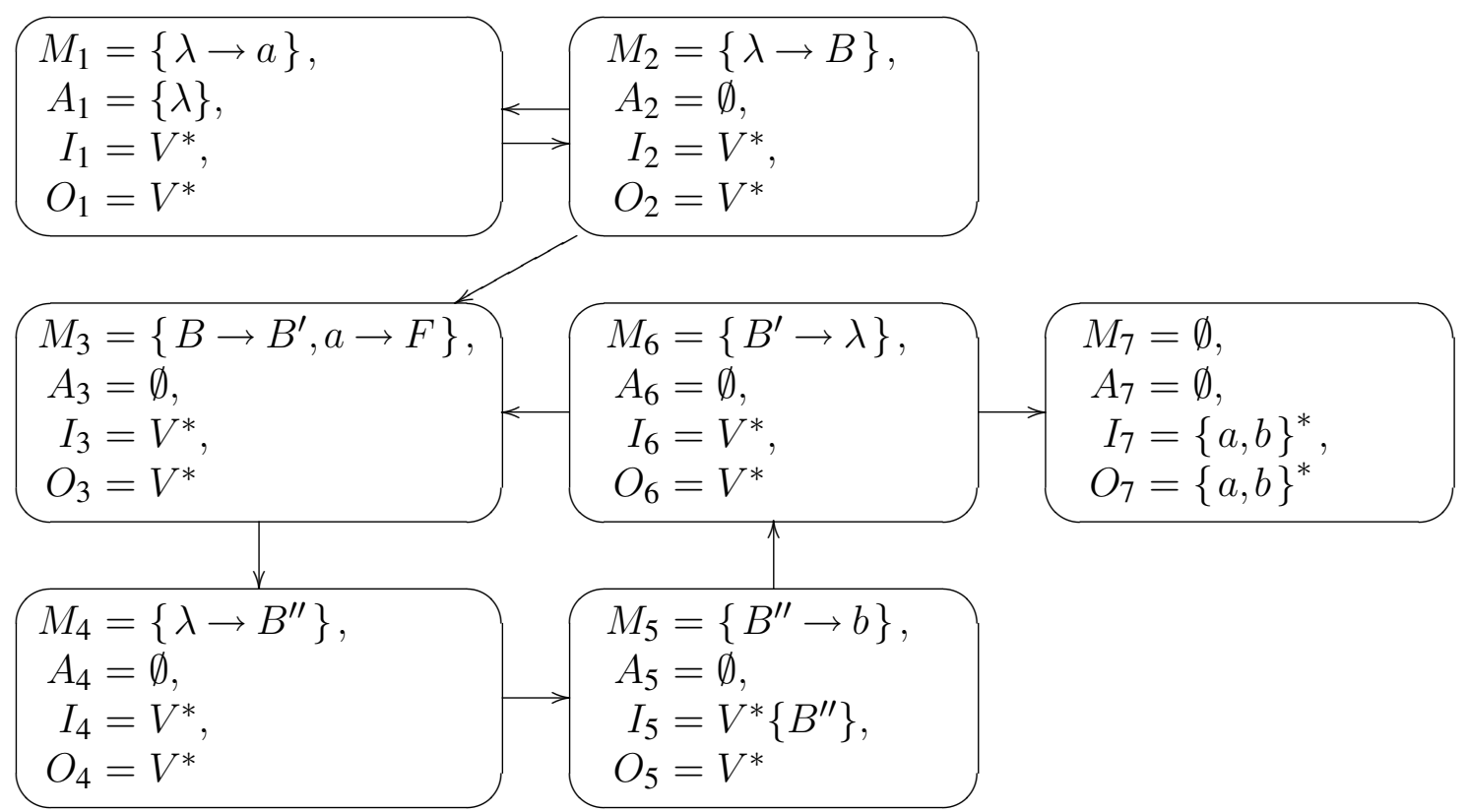

In the cycle consisting of $N_{1}$ and $N_{2}$, words are produced that have the same number of $a$ s and $B$ s. Whenever a word $w$ moves from $N_{2}$ to $N_{3}$ it satisfies $w \in\{a, B\}^{*}$ with $|w|_{a}=|w|_{B}=n$ where $n \geq 1$. In the cycle consisting of $N_{3}, N_{4}, N_{5}, N_{6}$, a $B$ is deleted and a $b$ is inserted at the end of the word. The word stays in this cycle until all $B$ s are consumed. In the end, the word is $a^{n} b^{n}$ in node $N_{5}$. This word enters the output node $N_{6}$ and also the node $N_{3}$. But there, only the rule $a \rightarrow F$ can be applied which introduces the trap symbol $F$ and the word remains in this node for ever.

Hence, $L(\mathcal{N})=L=\left\{a^{n} b^{n} \mid n \geq 1\right\}$ and $L \in \mathcal{E}(C O M B)$.

This language $L=\left\{a^{n} b^{n} \mid n \geq 1\right\}$ is a witness of the following proper inclusion.

Theorem 3.19 $\mathcal{E}(N I L) \subset \mathcal{E}(D E F)$.

Proof. The inclusion $\mathcal{E}(N I L) \subseteq \mathcal{E}(D E F)$ follows from Lemma 2.2. We now show that it is proper.

Let $L=\left\{a^{n} b^{n} \mid n \geq 1\right\}$. According to Lemma 3.18, $L \in \mathcal{E}(C O M B)$. Hence, also $L \in$ $\mathcal{E}(D E F)$ holds.

Let us assume that $L=L(\mathcal{N})$ for some evolutionary network $\mathcal{N}=\left(V, N_{1}, N_{2}, \ldots, N_{r}, E, j\right)$ with $N_{i}=\left(M_{i}, A_{i}, I_{i}, O_{i}\right)$ with a finite set $A_{i}$ and nilpotent filters $I_{i}$ and $O_{i}$ for $1 \leq i \leq r$. Then there is a natural number $s$ such that

$$
A_{i} \subseteq \bigcup_{k=0}^{s} V^{k}, \quad I_{i} \subseteq \bigcup_{k=0}^{s} V^{k} \text { or } I_{i}=I_{i}^{\prime} \cup V^{s} V^{+} \text {with } I_{i}^{\prime} \subseteq \bigcup_{k=0}^{s} V^{k}
$$


and

$$
O_{i} \subseteq \bigcup_{k=0}^{s} V^{k} \text { or } O_{i}=O_{i}^{\prime} \cup V^{s} V^{+} \text {with } O_{i}^{\prime} \subseteq \bigcup_{k=0}^{s} V^{k}
$$

for $1 \leq i \leq r$. Let

$$
D=w_{0} \Longrightarrow_{M_{q(1)}} w_{1} \vdash w_{1} \Longrightarrow_{M_{q(2)}} w_{2} \vdash w_{2} \Longrightarrow_{M_{q(3)}} w_{3} \vdash \cdots \Longrightarrow_{M_{q(p)}} w_{p} \vdash w_{p}
$$

be a derivation (the evolution steps are done according to the sets $M_{q(l)}, 1 \leq l \leq p$ ) and $w_{p}$ is contained in the output node.

We say that a letter $x$ is an $a$-occurrence (or $b$-occurrence or $\lambda$-occurrence) in $w_{t}, 1 \leq t \leq p$, if in all steps $w_{t+u} \Longrightarrow_{M_{q(t+u+1)}} w_{t+u+1}, u \geq 0$, the letter or its successors are not changed or changed by a substitution and the last change is a substitution of the form $z \rightarrow a$ (or $z \rightarrow b$ or $z \rightarrow \lambda$, respectively).

We say that $w_{t}$ is a Z-word if all steps $w_{t+u} \Longrightarrow_{M_{q(t+u+1)}} w_{t+u+1}, u \geq 0$, are substitutions or deletions or insertions of $\lambda$-occurrences.

Let now $w_{p}=a^{s} b^{s}$. Moreover, let $w_{l}$ be the first occurrence of a Z-word in $D$. By the definition of a Z-word, all words $w_{l}, w_{l+1}, \ldots, w_{p}$ have at least the length $2 s$. Thus, only infinite filters are used and if $w_{l+u}, u \geq 0$, passes such a filter, then any permutation of $w_{l+u}$ passes the filter, too. Moreover,

$$
w_{l}=c_{1} a_{1} c_{2} a_{2} \ldots c_{s} a_{s} c_{s+1} b_{1} c_{s+2} b_{2} \ldots c_{2 s} b_{s} c_{2 s+1}
$$

with several $a$-occurrences $a_{1}, a_{2}, \ldots, a_{s}$, several $b$-occurrences $b_{1}, b_{2}, \ldots, b_{s}$ and several (possibly empty) words $c_{1}, c_{2}, \ldots, c_{2 s+1}$ consisting of $\lambda$-occurrences. Furthermore, $w_{l-1} \Longrightarrow_{M_{q(l)}} w_{l}$ is an insertion of an $a$-occurrence or an insertion of a $b$-occurrence. Let us assume that $a_{f}$ or $b_{g}$ is inserted. Now we change the position of the insertion in the step $w_{l-1} \Longrightarrow_{M_{q(l)}} w_{l}$ such that

$$
w_{l-1} \Longrightarrow_{M_{q(l)}} c_{1} a_{1} c_{2} a_{2} \ldots c_{f-1} a_{f-1} c_{f} c_{f+1} a_{f+1} \ldots c_{s} a_{s} c_{s+1} b_{1} c_{s+2} b_{2} \ldots c_{2 s} b_{s} a_{f} c_{2 s+1}
$$

or

$$
w_{l-1} \Longrightarrow_{M_{q(l)}} c_{1} b_{g} a_{1} c_{2} a_{2} \ldots c_{s} a_{s} c_{s+1} b_{1} c_{s+2} b_{2} \ldots c_{s+g-1} b_{g-1} c_{s+g} c_{s+g+1} b_{g+1} \ldots c_{2 s} b_{s} a_{f} c_{2 s+1}
$$

is obtained. In the sequel, we perform the same operations as in $D$. Thus, we obtain finally $a^{s-1} b^{s} a$ or $b a^{s} b^{s-1}$ in the output node. Therefore

$$
a^{s-1} b^{s} a \in L(\mathcal{N}) \text { or } b a^{s} b^{s-1} \in L(\mathcal{N}),
$$

but

$$
a^{s-1} b^{s} a \notin L \text { and } b a^{s} b^{s-1} \notin L
$$

which contradicts $L=L(\mathcal{N})$.

Thus, $L \notin \mathcal{E}(N I L)$. Hence, we have a language in $\mathcal{E}(D E F) \backslash \mathcal{E}(N I L)$ and the proper inclusion $\mathcal{E}(N I L) \subset \mathcal{E}(D E F)$ is shown.

If the filters are combinational or reverse combinational languages, i. e., languages of the form $V^{*} A$ or $A V^{*}$, then every recursively enumerable language can be generated.

Theorem 3.20 $\mathcal{E}(B C O M B)=R E$. 
Proof. Let $L$ be a recursively enumerable language and $G=(N, T, P, S)$ be a grammar in Geffert normal form generating the language $L(G)=L$ with $N=\{S, A, B, C\}$. We construct a NEP $\mathcal{N}$ that simulates the derivation process in $G$ and, hence, generates the same language. The idea is to rotate the sentential form until the subword which has to be replaced is a suffix and then to delete and insert (if necessary) at the right end of the word.

Let $V=N \cup T$ and \# $\notin V$ be a symbol that marks the actual end of a word during the rotation. Let $U=V \cup\{\#\}, u=\#(U)$ be the number of the elements of $U$, and $x_{1}, x_{2}, \ldots, x_{u}$ be the elements of $U$. Additionally, let $U^{\prime}=\left\{x^{\prime} \mid x \in U\right\}$ and $\hat{U}=U \cup U^{\prime}$. Furthermore, let $p$ be the number of the rules of the form $S \rightarrow v$ and let these rules be $P_{i}=S \rightarrow a_{i, 1} a_{i, 2} \cdots a_{i, l_{i}}$ with $1 \leq i \leq p, l_{i} \geq 0$, and $a_{i, j} \in V$ for $1 \leq j \leq l_{i}$.

We construct subnetworks for rotating a word and for simulating the application of a rule. These subnetworks are connected by a special node (the 'master' $N_{0}$ ) that belongs to every subnetwork. The structure of the network can be seen in Figure 2.

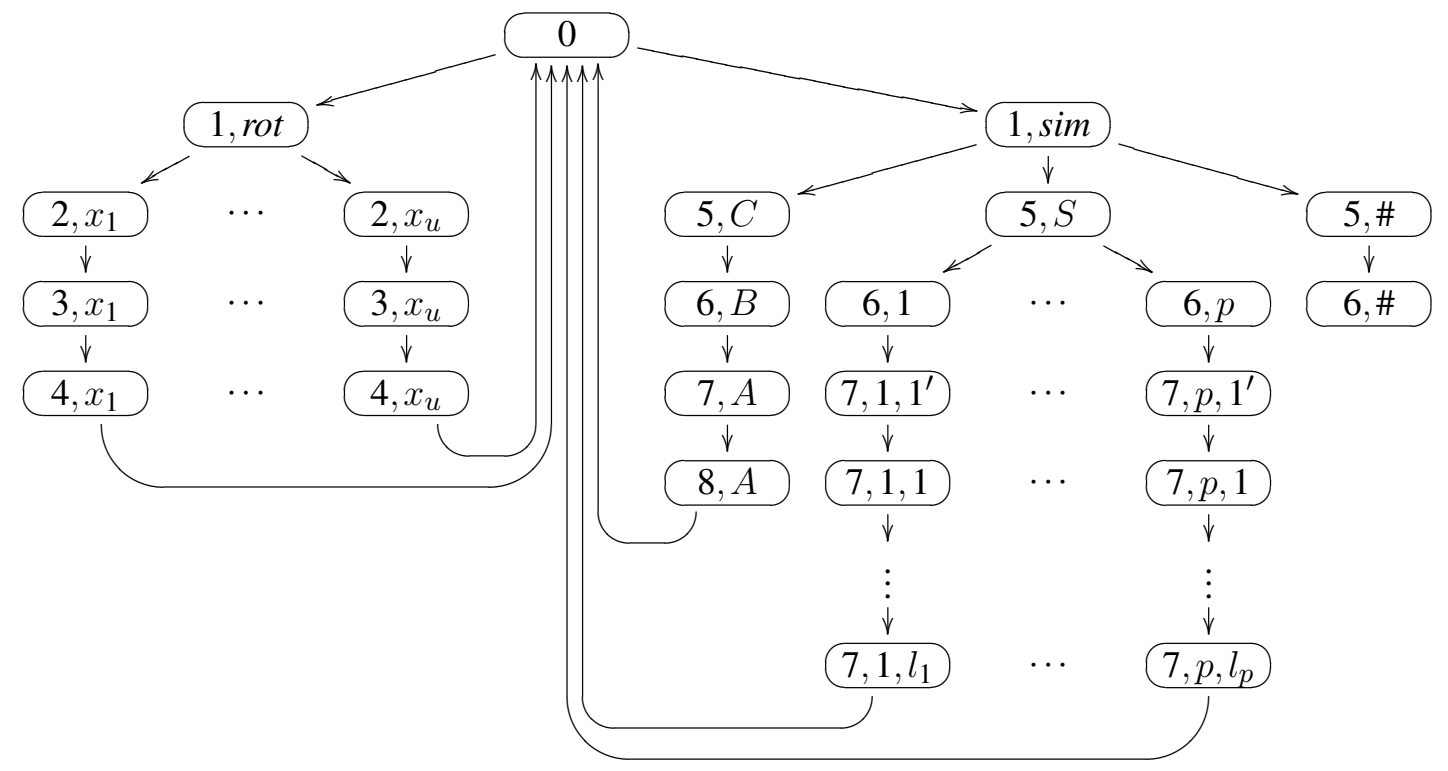

Figure 2: NEP for a Geffert normal form

The master $N_{0}$ is defined by $A_{0}=\{S \#\}, M_{0}=\emptyset, I_{0}=U^{+}$, and $O_{0}=U^{+}$. The work starts with the first sentential form of $G$ where the end is marked by \#. This node does nothing but in the next communication step the word will be sent to the nodes $N_{1, r o t}$ where the rotation will start and $N_{1, \text { sim }}$ where the simulation of a rule will start.

The initial languages $A_{i}$ of all other nodes $N_{i}$ are empty.

The node $N_{1, \text { rot }}$ is defined by $I_{1, \text { rot }}=U^{+}, M_{1, \text { rot }}=\left\{x \rightarrow x^{\prime} \mid x \in U\right\}$, and $O_{1, \text { rot }}=\hat{U}^{+}$. This node marks a symbol by changing it to a primed version. Then the word is sent to all nodes $N_{2, x}$ where $x \in U$. If another symbol than the left most one has been marked, the word leaves the network. If the left most symbol has been marked, then exactly one of these nodes gives access to the word, namely the node $N_{2, x}$ where $x$ is the symbol whose primed version stands at the beginning of the word. Thus, $N_{2, x}$ for $x \in U$ is defined by $I_{2, x}=\left\{x^{\prime}\right\} \hat{U}^{*}, M_{2, x}=\left\{x^{\prime} \rightarrow \lambda\right\}$, and $O_{2, x}=U^{+}$. The primed $x$ is deleted. Then the word is sent to the corresponding node $N_{3, x}$ where $x^{\prime}$ is inserted. Hence, $N_{3, x}$ for $x \in U$ is defined by $I_{3, x}=U^{+}, M_{3, x}=\left\{\lambda \rightarrow x^{\prime}\right\}$, and $O_{3, x}=\hat{U}^{+}$. Then the word is sent to node $N_{4, x}$ which takes the word only if $x^{\prime}$ is the 
last symbol (the right most symbol) of the word and replaces $x^{\prime}$ by $x$. Hence, $N_{4, x}$ for $x \in U$ is defined by $I_{4, x}=\hat{U}^{*}\left\{x^{\prime}\right\}, M_{4, x}=\left\{x^{\prime} \rightarrow x\right\}$, and $O_{4, x}=U^{+}$. Now, the word was rotated by one symbol and will be sent to the master.

As mentioned above, the simulation of a rule will start in the node $N_{1, \text { sim }}$. It is defined by $I_{1, \text { sim }}=U^{+}, M_{1, \text { sim }}=\left\{x \rightarrow x^{\prime} \mid x \in U\right\}$, and $O_{1, \text { sim }}=\hat{U}^{+}$. In this node, a symbol will be changed to the primed version. If another symbol than the right most one is primed, the word is lost in the next communication step. If the last symbol was $C$ and the rule $A B C \rightarrow \lambda$ exists in $P$ then this rule can be startet to be simulated (maybe $C$ is not preceeded by $A B$ then the simulation does not work which will be noticed later). If the last symbol was $S$ then one of the rules $P_{i}(1 \leq i \leq p)$ can be simulated. If the last symbol was \# then this end marker should be removed to obtain a real sentential form of $G$ (which is the end of the simulation of $G$ ). In all other cases, we do not need the word anymore.

We now construct subnetworks for these three cases.

If the erasing rule $A B C \rightarrow \lambda$ exists in $P$ then we define the following nodes: $N_{5, C}$ is defined by $I_{5, C}=\hat{U}^{*}\left\{C^{\prime}\right\}, M_{5, C}=\left\{C^{\prime} \rightarrow \lambda\right\}$, and $O_{5, C}=U^{+}$. This node takes a word if the last symbol is $C^{\prime}$, it deletes this symbol and passes on the word to the next node $N_{6, B}$ which is responsible for the $B$. The node $N_{6, B}$ is defined by $I_{6, C}=U^{*}\{B\}, M_{6, B}=\{B \rightarrow \lambda\}$, and $O_{6, B}=U^{+}$. This node takes the word if the last symbol is $B$, otherwise the word is lost because the previously selected $C$ does not belong to a subword $A B C$ and therefore the rule cannot be applied. The node $N_{6, B}$ deletes a $B$, and sends the word to node $N_{7, A}$ defined by $I_{7, A}=U^{*}\{A\}, M_{7, A}=\left\{A \rightarrow A^{\prime}\right\}$, and $O_{7, A}=\hat{U}^{+}$. If $N_{6, B}$ did not delete the last symbol, then the word will be lost since an $A$ is expected now. If the last symbol of the word received is $A$ then the simulation continues, otherwise the word is lost (the previously selected $C$ does not belong to a subword $A B C$ and therefore the rule cannot be applied). In $N_{7, A}$ the symbol $A$ is marked. The word moves to the node $N_{8, A}$ defined by $I_{8, A}=\hat{U}^{*}\left\{A^{\prime}\right\}, M_{8, A}=\left\{A^{\prime} \rightarrow \lambda\right\}$, and $O_{8, A}=U^{+}$. This node ensures that the $A$ in the end is deleted and not somewhere else. Then a word $u \# v$ has been derived by simulating the rule $A B C \rightarrow \lambda$ where $v u$ is a sentential form of $G$. The word $u \# v$ is sent to the master node $N_{0}$.

For the rules of the form $S \rightarrow v$, we define an initial node $N_{5, S}$ that prepares the simulation. Let $N_{5, S}$ be defined by the input filter $I_{5, S}=\hat{U}^{*}\left\{S^{\prime}\right\}$, the set $M_{5, S}=\left\{S^{\prime} \rightarrow S_{i} \mid 1 \leq i \leq p\right\}$, and the output filter $O_{5, S}=\left(U \cup\left\{S_{i} \mid 1 \leq i \leq p\right\}\right)^{+}$. This node chooses a rule $P_{i}$ that is simulated afterwards. The word obtained is sent to all nodes $N_{6, j}$ with $1 \leq j \leq p$ but it is accepted only by node $N_{6, i}$ which corresponds to the rule selected.

For each rule $P_{i}$ with $1 \leq i \leq p$, we define the following nodes: the node $N_{6, i}$ is defined by $I_{6, i}=\left(\left\{S_{i}\right\} \cup U\right)^{+}, M_{6, i}=\left\{S_{i} \rightarrow \lambda\right\}$, and $O_{6, i}=U^{+}$. This node deletes $S_{i}$ (the symbol corresponding to the left hand side of the rule under consideration). If $P_{i}=S \rightarrow \lambda$ then this word is sent back to the master node, otherwise the rule is $S \rightarrow a_{i, 1} a_{i, 2} \cdots a_{i, l_{i}}$ for a natural number $l_{i} \geq 1$ and symbols $a_{i, j} \in V$ for $1 \leq j \leq l_{i}$. These symbols will now be appended to the word in a chain of nodes (two nodes for each symbol, one for inserting and one for testing whether the insertion took place in the end of the word). For each number $j$ with $1 \leq j \leq l_{i}$, we define the nodes $N_{7, i, j^{\prime}}$ by $I_{7, i, j^{\prime}}=U^{+}, M_{7, i, j^{\prime}}=\left\{\lambda \rightarrow a_{i, j}^{\prime}\right\}$, and $O_{7, i, j}=\hat{U}^{+}$as well as $N_{7, i, j}$ by $I_{7, i, j}=\hat{U}^{*}\left\{a_{i, j}^{\prime}\right\}, M_{7, i, j}=\left\{a_{i, j}^{\prime} \rightarrow a_{i, j}\right\}$, and $O_{7, i, j}=U^{+}$. After the right hand side of the rule $P_{i}$ is appended, the rule has been simulated, and the word is sent to the master node. 
For the case that the marked symbol in $N_{1, \text { sim }}$ is the last symbol and the end marker \#, we define the following nodes: $N_{5, \#}$ by $I_{5, \#}=U^{*}\left\{\#^{\prime}\right\}, M_{5, \#}=\left\{\#^{\prime} \rightarrow \lambda\right\}$, and $O_{5, \#}=U^{*}$, as well

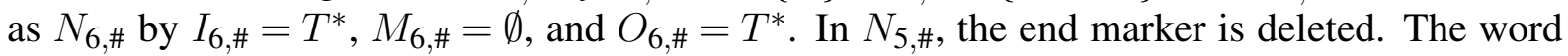

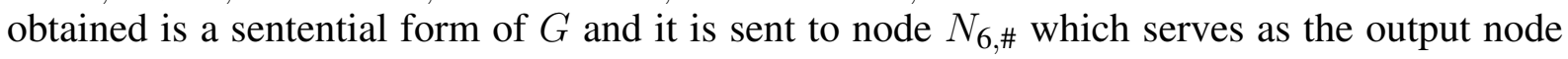
and takes the word only if it is a terminal word.

The NEP $\mathcal{N}$ is defined as

$$
\mathcal{N}=\left(X, N_{0}, \ldots, N_{6, \#}, E, N_{6, \#}\right)
$$

with the working alphabet $X=U \cup U^{\prime} \cup\left\{S_{i} \mid 1 \leq i \leq p\right\}$, the nodes defined above, the set $E$ of

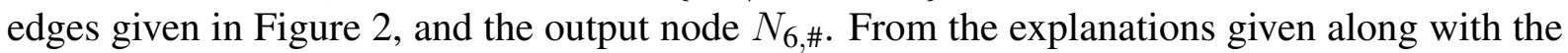
definitions of the nodes and edges, it follows that the network $\mathcal{N}$ generates the same language as the grammar $G: L(\mathcal{N})=L$. Moreover, all filters are sets of the form $A Y^{*}$ or $Y^{*} A$ for some alphabet $Y$ and $A \subseteq Y$. Hence, $R E \subseteq \mathcal{E}(B C O M B)$. With Theorem 2.3, $\mathcal{E}(B C O M B)=R E$ follows.

Completeness can also be achived if all filters are ordered. Then the completeness follows for networks where all filters are non-counting or all are power-separating.

Theorem 3.21 $\mathcal{E}(O R D)=\mathcal{E}(N C)=\mathcal{E}(P S)=R E$.

Proof. By Lemma 2.2, the inclusions shown in Figure 1, and Theorem 2.3, it is sufficient to prove that $R E \subseteq \mathcal{E}(O R D)$.

Consider the network constructed in the proof of Theorem 3.20 . All filters are languages of the form $V^{*}$ or $V^{*} A$ or $A V^{*}$ and can be accepted by deterministic finite automata with one state or the following transition graphs:
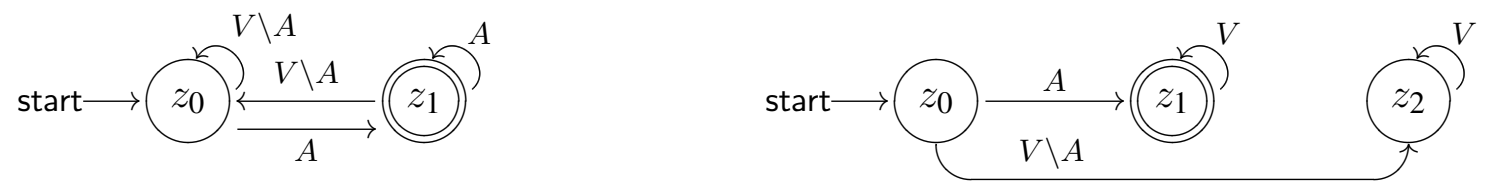

These automata have transition functions $\delta_{C O M B}$ and $\delta_{B C O M B}$ given by the following tables:

\begin{tabular}{|c|c|c|}
\hline$\delta_{\text {СОMB }}$ & $a \in A$ & $a \in V \backslash A$ \\
\hline$z_{0}$ & $z_{1}$ & $z_{0}$ \\
$z_{1}$ & $z_{1}$ & $z_{0}$ \\
& & \\
\hline
\end{tabular}

\begin{tabular}{|c|c|c|}
\hline$\delta_{\text {BСОMB }}$ & $a \in A$ & $a \in V \backslash A$ \\
\hline$z_{1}$ & $z_{1}$ & $z_{1}$ \\
$z_{0}$ & $z_{1}$ & $z_{2}$ \\
$z_{2}$ & $z_{2}$ & $z_{2}$ \\
\hline
\end{tabular}

It is to be seen that the states in each column for $A$ and $V \backslash A$ preserve the order given in the first column. Hence, all filters are ordered languages.

It remains open whether also definite or even combinational filters are sufficient for generating all recursively enumerable languages. We conjecture that the use of combinational filters is as powerful as the use of definite filters. 


\section{Conclusion}

The results obtained can be seen in Figure 3

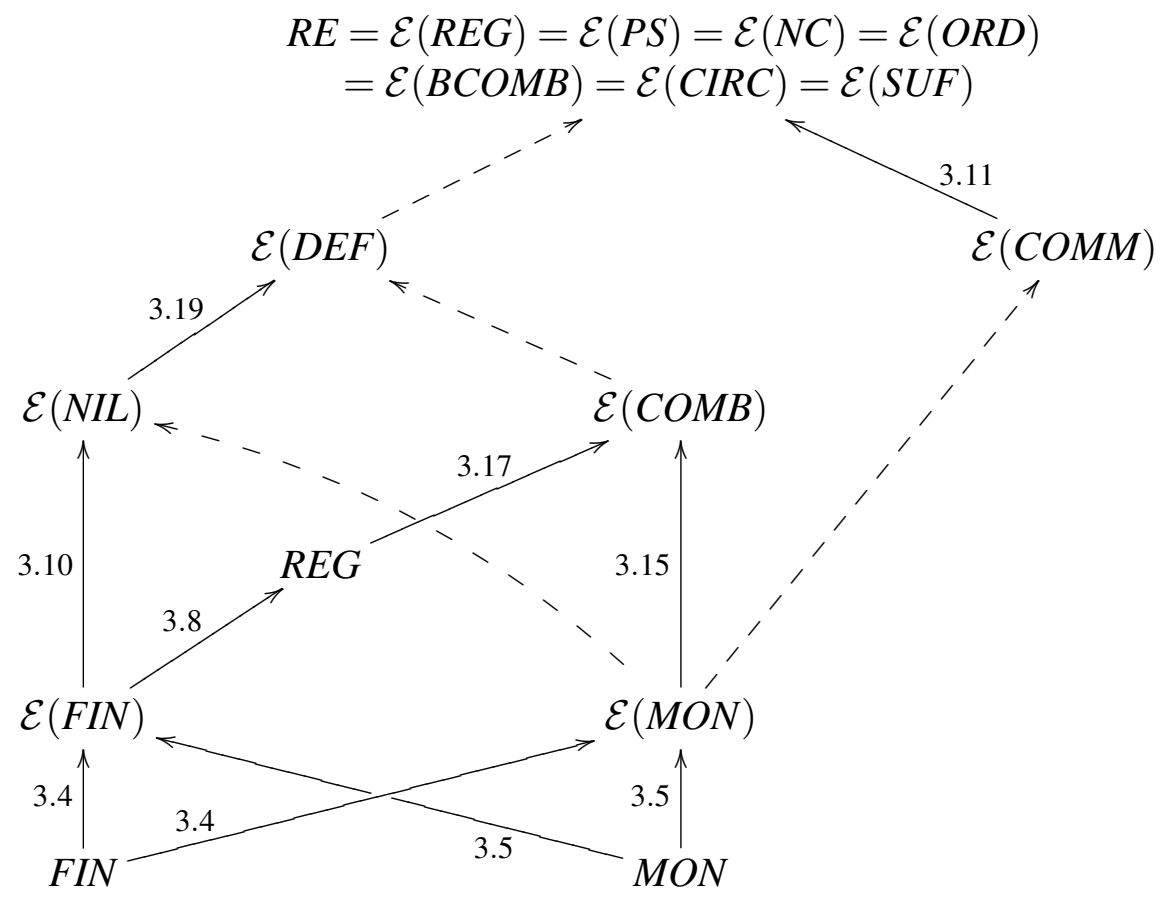

Figure 3: Hierarchy of the language families generated by NEPs with subregular filters

A solid arrow from $X$ to $Y$ denotes $X \subset Y$ ( $X$ is a proper subset of $Y$ ), a dashed arrow from $X$ to $Y$ denotes $X \subseteq Y$; if two families are not connected by a directed path then they are not necessarily incomparable. The number at an arrow is the number of the theorem which states the respective inclusion.

We continue the investigations in order to find out whether or not the remaining inclusions are proper and to characterize the families of languages. Especially, regarding the classes $\mathcal{E}(C O M B)$ and $\mathcal{E}(D E F)$, we have the conjucture and ideas for a proof that they coincide

\section{References}

[1] A. Alhazov, E. Csuhaj-Varjú, C. Martín-Vide, Y. Rogozhin, On the size of computationally complete hybrid networks of evolutionary processors. Theoretical Computer Science 410 (2009), 3188-3197.

[2] A. Alhazov, J. Dassow, C. Martín-Vide, Y. Rogozhin, B. Truthe, On networks of evolutionary processors with nodes of two types. Fundamenta Informaticae 91 (2009), $1-15$.

[3] J. Castellanos, C. Martín-Vide, V. Mitrana, J. M. Sempere, Solving NPComplete Problems With Networks of Evolutionary Processors. In: IWANN '01: Proceedings of the 6th International Work-Conference on Artificial and Natural Neural Networks, LNCS 2084. Springer-Verlag Berlin, 2001, 621-628. 
[4] J. Castellanos, C. Martín-Vide, V. Mitrana, J. M. Sempere, Networks of Evolutionary Processors. Acta Informatica 39 (2003) 6-7, 517-529.

[5] E. Csuhaj-Varjú, V. Mitrana, Evolutionary systems: A language generating device inspired by evolving communities of cells. Acta Informatica 36 (2000) 11, 913-926.

[6] E. Csuhaj-Varjú, A. SalomaA, Networks of Parallel Language Processors. In: New Trends in Formal Languages - Control, Cooperation, and Combinatorics (to Jürgen Dassow on the occasion of his 50th birthday). LNCS 1218, Springer-Verlag Berlin, 1997, 299-318.

[7] L. D. ERrico, C. Jesshope, Towards a new architecture for symbolic processing. In: AIICSR'94: Proceedings of the sixth international conference on Artificial intelligence and information-control systems of robots. World Scientific Publishing Co., Inc., River Edge, NJ, USA, 1994, 31-40.

[8] S. E. Fahlman, G. E. Hinton, T. J. Sejnowski, Massively parallel architectures for AI: NETL, Thistle, and Boltzmann machines. In: Proc. AAAI 1983. 1983, 109-113.

[9] V. GEFFERT, Normal forms for phrase-structure grammars. RAIRO - Theoretical Informatics and Applications 25 (1991), 473-496.

[10] I. M. HAVEL, The theory of regular events II. Kybernetika 5 (1969) 6, 520-544.

[11] W. D. Hillis, The Connection Machine. MIT Press, Cambridge, MA, USA, 1986.

[12] C. Martín-Vide, V. Mitrana, Networks of evolutionary processors: Results and perspectives. In: Molecular Computational Models: Unconvent. Approaches. 2005, 78-114.

[13] G. PĂUn, Computing with membranes. J. Comput. Syst. Sci. 61 (2000) 1, 108-143.

[14] G. PĂUn, L. SÂntean, Parallel communicating grammar systems: The regular case. Annals of University of Bucharest, Ser. Matematica-Informatica 38 (1989), 55-63.

[15] G. Rozenberg, A. SalomaA, Handbook of Formal Languages. Springer-Verlag, Berlin, 1997.

[16] A. SalomaA, Formal Languages. Springer-Verlag, Berlin, 1978.

[17] D. Sankoff, G. Leduc, N. Antoine, B. Paquin, F. Lang, R. Cedergren, Gene order comparisons for phylogenetic inference: Evolution of the mitochondrial genome. Proceedings of the National Academy of Sciences of the United States of America 89 (1992) 14, 6575-6579.

[18] B. Wiedemann, Vergleich der Leistungsfähigkeit endlicher determinierter Automaten. Diplomarbeit, Universität Rostock, 1978. 